\title{
A VIRO THEOREM WITHOUT CONVEXITY HYPOTHESIS FOR TRIGONAL CURVES
}

\author{
Benoit Bertrand $\quad$ Erwan Brugallé
}

\begin{abstract}
A cumbersome hypothesis for Viro patchworking of real algebraic curves is the convexity of the given subdivision. It is an open question in general to know whether the convexity is necessary. In the case of trigonal curves we interpret Viro method in terms of dessins d'enfants. Gluing the dessins d'enfants in a coherent way we prove that no convexity hypothesis is required to patchwork such curves.
\end{abstract}

\section{Introduction}

In the late seventies O. Ya. Viro invented a powerful method to construct real algebraic hypersurfaces with prescribed topology in toric varieties. Since then it has become the main tool to prove the existence of certain topological types among families of real algebraic varieties.

Classification of the real schemes realized by nonsingular curves of degree 7 in $\mathbb{R} P^{2}$ (Vir84]), smoothing of curves with complicated singularities (Vir89], Shu99]), maximal curvature of plane algebraic curves ([LdM]), construction of counterexamples to the Ragsdale conjecture ([te93, Ite01, Haa95], [San and [Bru06]) and construction of projective hypersurfaces with big Betti numbers ([Bih] are examples of results using Viro method. It has also been used to prove maximality results. A real algebraic variety $X$ is said to be maximal ( $M$-variety) if the sum of the Betti numbers with $\mathbb{Z}_{2}$ coefficients of its real part is equal to the corresponding sum for its complexification $\left(b_{*}\left(\mathbb{R} X ; \mathbb{Z}_{2}\right)=b_{*}\left(\mathbb{C} X ; \mathbb{Z}_{2}\right)\right)$. Viro method has been very useful to prove for instance the existence of projective $M$-hypersurfaces of any degree in any dimension (IV]) or existence of asymptotically maximal families of hypersurfaces in any projective toric variety (Ber06).

Roughly speaking, the Viro method (for curves) can be described as follows : start with some real algebraic curves $\left(C_{i}\right)$ whose Newton polygons are the 2-cells of a polygonal subdivision $\tau$ of a convex polygon $\Delta$. Then, under some assumptions on the $C_{i}$ 's and on $\tau$, the Viro Theorem asserts that there exists a real algebraic curve with Newton polygon $\Delta$ obtained as a topological gluing of the curves $C_{i}$. One of the hypothesis of Viro's theorem is that the subdivision $\tau$ should be convex, i.e. there should exist a convex piecewise-linear affine function $\lambda: \Delta \rightarrow \mathbb{R}$ whose domains of linearity are exactly the Newton polygons of the curves $C_{i}$. Notice that non-convex subdivisions do exist (see CH80), an example is depicted in Figure 2

It is an open problem to know whether the convexity hypothesis is necessary or not. More precisely it is not known whether a curve patchworked from a non-convex subdivision is isotopic to a real algebraic curve with Newton polygon $\Delta$ (see dLW98 and IS02]). I. Itenberg and E. Shustin proved in IS02 that in the case of $\mathbb{C} P^{2}$ or of the geometrically ruled rational surfaces $\Sigma_{n}$ there always exists

Both authors are very grateful to the Max Planck Institute für Mathematik in Bonn for its financial support and excellent working conditions.

2000 Mathematics Subject Classification : 14P25

Key words : topology of real algebraic curves, Viro method, dessin d'enfants, rational ruled surfaces 
a real pseudoholomorphic curve isotopic to the patchworked one. Note that it is still unknown if there exists a real nonsingular pseudoholomorphic curve in $\mathbb{R} P^{2}$ which is not isotopic to a real nonsingular algebraic curve of the same degree.

If the convexity hypothesis in the Viro method turned out to be necessary in general it could lead to such examples of "non-algebraic pseudoholomorphic curves". On the other hand if the convexity hypothesis could be removed it would simplify greatly the Viro construction since it is often rather technical to check whether a given subdivision is convex. Moreover this approach would give a deeper insight or at least a new interpretation of the Viro method. This work is a first step in the study of the need of the convexity hypothesis.

Trigonal curves are those curves in $\Sigma_{n}$ whose Newton polygon is the triangle $\Delta_{n}$ with vertices $(0,0)$, $(0,3)$ and $(3 n, 0)$. In this article we prove that we can get rid of the convexity hypothesis for trigonal curves. Namely, any curve patchworked from $\Delta_{n}$ is an algebraic trigonal curve in $\Sigma_{n}$ (with Newton polygon $\Delta_{n}$ ). In fact we construct curves with prescribed positions with respect to the natural pencil of lines of $\Sigma_{n}$. In the convex case, the pencil of lines has been studied by L. Lopez de Medrano in [LdM].

We want to construct a trigonal curve $C$ out of a trigonal patchwork. The strategy is the following. We first cut $C$ in several pieces which are the intersection of $C$ with some portions of the pencil of lines in $\Sigma_{n}$. All these pieces are given by the subdivision of $\Delta_{n}$ and will be algebraic. In [S02, Itenberg and Shustin glue these pieces topologically in order to obtain a real pseudoholomorphic curve.

Since we deal with trigonal curve we are able to use a particular case of "dessins d'enfants" which are called real rational graphs. This technic was introduced in real algebraic geometry independently by S. Yu. Orevkov in Ore03 and by S. Natanzon, B. Shapiro and A. Vainshtein in NSV02. The position of a real trigonal curve with respect to the pencil in $\Sigma_{n}$ is encoded by three linearly dependent real polynomials (see Ore03 or section 4). Real rational graphs give a necessary and sufficient condition for the existence of three linearly dependent real polynomials in one variable whose roots realize a given real arrangement. Via Riemann existence theorem they provide an existence criterion for a given trigonal curve.

In fact, we will use a slight generalization of these objects. To glue algebraically the pieces of our curve we indeed need to perform some surgery on dessins d'enfants enhanced with the data of the sign of some characteristic polynomials. These are what we call signed real rational graphs. Once glued together in a coherent way the signed real rational graphs obtained for each piece yield a dessin d'enfant which corresponds to a curve with the required topology.

\section{Organization of this article}

In section 2 we recall some facts about rational geometrically ruled surfaces. In section 3 we explain the patchwork construction and give a patchwork theorem for real algebraic trigonal curves without any convexity assumption (Theorem 3.4). We explain in section 4 how to encode the topology of a trigonal curve in a sign array. In section 5 we define an order on the polygons of a patchwork which is used in section [6 to extract a sign array from a trigonal patchwork. We state there our main result (Theorem 6.2) . The rest of the paper is devoted to the proof of this theorem. The main tool of this proof, signed real rational graphs, is defined in section [7. In section 8 we associate a signed real rational graph to each piece of the pencil of line given by the order on the polygons of a patchwork. We glue all these graph in section 9 and check in section 10 that the obtained signed real rational graph corresponds to a real algebraic trigonal curve which has the topology prescribed by the patchwork. 


\section{Notation}

All polynomials in two variables $C(X, Y)$ are considered as polynomials in the variable $Y$ whose coefficients are polynomials in the variable $X$. By the discriminant of $C(X, Y)$ we mean the discriminant of $C(X, Y)$ with respect to the variable $Y$.

Suppose that a coordinate system of $\mathbb{R} P^{1}$ is fixed. The point $[0: 1]$ (resp. [1:0]) is denoted by 0 $($ resp. $\infty)$, and the points $[x: y]$ such that $x y>0$ (resp. $x y<0)$ are called positive (resp. negative). The embedding of $\mathbb{R}$ into $\mathbb{R} P^{1}$ given by $x \mapsto[x: 1]$ induces an orientation of $\mathbb{R} P^{1}$. Given two points $A$ and $B$ in $\mathbb{R} P^{1}$, the segment $[A ; B]$ is the connected component of $\mathbb{R} P^{1} \backslash\{A, B\}$ oriented from $A$ to $B$ for this orientation.

When there is no ambiguity, the two words "curve" and "polynomial" are used to designate either a polynomial $C(X, Y)$ or the curve defined by this polynomial.

\section{Rational geometrically ruled surfaces}

The $n^{\text {th }}$ rational geometrically ruled surface, denoted by $\Sigma_{n}$, is the surface obtained by taking four copies of $\mathbb{C}^{2}$ with coordinates $(x, y),\left(x_{2}, y_{2}\right),\left(x_{3}, y_{3}\right)$ and $\left(x_{4}, y_{4}\right)$, and by gluing them along $\left(\mathbb{C}^{*}\right)^{2}$ with the identifications $\left(x_{2}, y_{2}\right)=\left(1 / x, y / x^{n}\right),\left(x_{3}, y_{3}\right)=(x, 1 / y)$ and $\left(x_{4}, y_{4}\right)=\left(1 / x, x^{n} / y\right)$. Let us denote by $E$ (resp. $B$ and $F$ ) the algebraic curve in $\Sigma_{n}$ defined by the equation $\left\{y_{3}=0\right\}$ (resp. $\{y=0\}$ and $\{x=0\})$. The coordinate system $(x, y)$ is called standard. The projection $\pi:(x, y) \mapsto x$ on $\mathbb{C}^{2}$ defines a $\mathbb{C}^{1}$-bundle structure on $\mathbb{C}^{2}$ which is extendable up to a $\mathbb{C} P^{1}$-bundle structure on $\Sigma_{n}$. The intersection numbers of $B$ and $F$ are respectively $B \circ B=n, F \circ F=0$ and $B \circ F=1$. The surface $\Sigma_{n}$ has a natural real structure induced by the complex conjugation in $\mathbb{C}^{2}$, and the real part $\mathbb{R} \Sigma_{n}$ of $\Sigma_{n}$ is a torus if $n$ is even and a Klein bottle if $n$ is odd. The restriction of $\pi$ on $\mathbb{R} \Sigma_{n}$ defines a pencil of lines denoted by $\mathcal{L}$.

The group $H_{2}\left(\Sigma_{n}, \mathbb{Z}\right)$ is isomorphic to $\mathbb{Z} \times \mathbb{Z}$ and is generated by the classes of $B$ and $F$. Moreover, one has $E=B-n F$. An algebraic curve on $\Sigma_{n}$ is said to be of bidegree $(k, l)$ if it realizes the homology class $k B+l F$ in $H_{2}\left(\Sigma_{n}, \mathbb{Z}\right)$. Its equation in $\Sigma_{n} \backslash E$ is

$$
\sum_{i=0}^{k} a_{k-i}(X, Z) Y^{i}
$$

where $a_{j}(X, Z)$ is a homogeneous polynomial of degree $n j+l$. A curve of bidegree $(3,0)$ is called $a$ trigonal curve on $\Sigma_{n}$.

In the rational geometrically ruled surfaces, we study real curves up to isotopy with respect to $\mathcal{L}$. Two curves are said to be isotopic with respect to the fibration $\mathcal{L}$ if there exists an isotopy of $\mathbb{R} \Sigma_{n}$ which transforms the first curve to the second one, and which maps each line of $\mathcal{L}$ to another line of $\mathcal{L}$. In this paper, curves in a rational geometrically ruled surface are depicted up to isotopy with respect to $\mathcal{L}$.

Definition 2.1 An algebraic curve $C$ of bidegree $(k, l)$ in $\Sigma_{n}$ is said to be $\mathcal{L}$-nonsingular if $C$ is nonsingular and if for any fiber $F$ in $\Sigma_{n}$, the set $F \cap C$ contains at least $k-1$ points.

\section{Patchworking real algebraic trigonal curves}

Here, we explain the patchworking method for curves. The general patchworking Theorem, which uses the convexity of the subdivision, can be found in Vir84, Vir89, Vir, Ris92 and [S03. Our 
patchwork Theorem, Theorem 3.4 does not require this convexity assumption, yet it works only for trigonal curves.

\subsection{Chart of a real polynomial}

This is the key notion of the Viro method. A chart of a real polynomial $C(X, Y)$ is a way to draw the curve defined by $C$ in $\left(\mathbb{R}^{*}\right)^{2}$ in the union of 4 symmetric copies of its Newton polygon. The Newton polygon of a polynomial $F$ will be denoted by $\Delta(F)$.

Given a convex polygon $\Delta$ in $\mathbb{R}^{2}$ with vertices in $\mathbb{Z}^{2}$, one can define the so called moment map from $\left(\mathbb{R}_{+}^{*}\right)^{2}$ to the interior of $\Delta$ as follows

$$
\begin{aligned}
\mu_{\Delta}:\left(\mathbb{R}_{+}^{*}\right)^{2} & \rightarrow \quad \\
(x, y) & \mapsto \frac{\sum_{(i, j) \in \Delta \cap \mathbb{Z}^{2} x^{i} y^{j} .(i, j)}}{\sum_{(i, j) \in \Delta \cap \mathbb{Z}^{2}} x^{i} y^{j}}
\end{aligned}
$$

If $\operatorname{dim}(\Delta)=2$, then $\mu_{\Delta}$ is a diffeomorphism. For a pair $\left(\epsilon_{1}, \epsilon_{2}\right) \in\{+,-\}^{2}$, we denote by $s_{\epsilon_{1}, \epsilon_{2}}$ the symmetry of $\mathbb{R}^{2}$ given by $s_{\epsilon_{1}, \epsilon_{2}}(x, y)=\left(\epsilon_{1} x, \epsilon_{2} y\right)$. For a convex polygon $\Delta$ in $\left(\mathbb{R}_{+}\right)^{2}$, we define $\Delta^{*}$ as $\bigcup_{\left(\epsilon_{1}, \epsilon_{2}\right) \in\{+,-\}^{2}} s_{\epsilon_{1}, \epsilon_{2}}(\Delta)$.

Definition 3.1 Let $C(X, Y)$ be a real polynomial. The chart of $C(X, Y)$, denoted by $C h(C)$, is the closure of the set

$$
\bigcup_{\left(\epsilon_{1}, \epsilon_{2}\right) \in\{0,1\}^{2}} s_{\epsilon_{1}, \epsilon_{2}} \circ \mu_{\Delta(C)} \circ s_{\epsilon_{1}, \epsilon_{2}}\left(\left\{(x, y) \in \mathbb{R}_{\epsilon_{1}}^{*} \times \mathbb{R}_{\epsilon_{2}}^{*} \mid C(x, y)=0\right\}\right)
$$

It is clear that $C h(C) \subset \Delta^{*}$.

\subsection{Gluing of charts}

Let $C(X, Y)=\sum a_{i, j} X^{i} Y^{j}$ be a polynomial and $\gamma$ be a face of $\Delta(C)$.

Definition 3.2 The truncation of $C(X, Y)$ on $\gamma$ is the polynomial $\sum_{(i, j) \in \gamma} a_{i, j} X^{i} Y^{j}$.

The polynomial $C(X, Y)$ is totally nondegenerate if the truncation of $C$ to any face of $\Delta(C)$ is nonsingular in $\left(\mathbb{C}^{*}\right)^{2}$.

A patchwork is a way to glue together the charts of real algebraic curves.

Definition 3.3 Let $\Delta$ be a convex polygon with vertices in $\mathbb{Z}^{2}$. A patchwork with support $\Delta$ is a pair $\left(\tau,\left(C_{i}(X, Y)\right)_{1 \leq i \leq r}\right)$ such that

- $\tau$ is a subdivision of $\Delta$,

- the $\left(C_{i}(X, Y)\right)$ are real algebraic curves,

- for any 2-cell of $\tau$, there is one and only one curve $C_{i}$ whose Newton polygon is this polygon,

- for any edge $\gamma$ of $\tau$, the truncations on $\gamma$ of two polynomials whose Newton polygon contains $\gamma$ coincide.

If $\Delta$ is the triangle with vertices $(0,0),(0,3)$ and $(3 n, 0)$ and if the coefficient of $Y^{3}$ of any curve $C_{i}(X, Y)$ is either 0 or 1 , we say that the patchwork is trigonal of degree $n$.

As we deal with nonsingular curves, we will consider patchworks satisfying the following condition 
(1) Each of the polynomials $C_{i}(X, Y)$ is totally nondegenerate.

The next theorem is a corollary of Theorem 6.2 and will be proved in section 6 ,

Theorem 3.4 Let $\Pi$ be a trigonal patchwork which satisfies hypothesis (1) above. Then there exists a real algebraic trigonal curve in $\Sigma_{n}$ whose chart is isotopic to $\cup_{1 \leq i \leq r} C h\left(C_{i}\right)$.

Through all this paper, we will consider trigonal patchworks which satisfy one more condition. This condition is due to the fact that we construct curves which have a generic position with respect to the pencil of lines. This condition is a technical ingredient in the proof of Theorem 3.4. One can always perturb slightly the curves involved in a patchwork so that they satisfy this condition, hence it does not appear in the statement of Theorem 3.4 .

(2) for any $i \in\{1, \ldots, r\}$, the curves $C_{i}(X, Y)$ and $\frac{\partial C_{i}}{\partial Y}(X, Y)$ have the maximal number of intersection points in $\left(\mathbb{C}^{*}\right)^{2}$ among curves with fixed Newton polygon $\Delta\left(C_{i}\right)$.

Remark : The last condition simply says that the curve has only ordinary tangency points with the vertical pencil of lines in $\left(\mathbb{C}^{*}\right)^{2}$ and that none of these points lie on a toric divisor corresponding to a horizontal edge in the toric surface associated to $\Delta\left(C_{i}\right)$.

We point out a consequence of condition (2) : suppose that $C_{i}(X, Y)=a_{j}(X) Y^{k}+a_{j-1} Y^{k+1}+$ $a_{j-2}(X) Y^{k+2} \ldots+a_{j-l}(X) Y^{k+l}$ and that $a_{j}\left(x_{0}\right)=0$ (resp. $a_{j-l}\left(x_{0}\right)=0$ ) with $x_{0} \in \mathbb{R}^{*}$, then $a_{j-1}\left(x_{0}\right) \neq 0\left(\right.$ resp. $\left.a_{j-l+1}\left(x_{0}\right) \neq 0\right)$.

\section{The sign array of a real algebraic trigonal curve}

\subsection{Polynomials of degree 3 in one variable}

Let us first consider a real polynomial of degree 3 in one variable $C(Y)=Y^{3}+a_{1} Y^{2}+a_{2} Y+a_{3}$. It is well known that the following three numbers play an important role in the resolution of such an equation

$$
P=a_{2}-\frac{a_{1}^{2}}{3}, \quad Q=a_{3}-\frac{a_{2} a_{1}}{3}+\frac{2 a_{1}^{3}}{27}, \quad D=-4 P^{3}-27 Q^{2} .
$$

The number $D$ is the discriminant of the polynomial $C$. In particular one has

- if $D>0$, then $C$ has three distinct real roots,

- if $D<0$, then $C$ has one real root and two complex conjugated non-real roots,

- if $D=0$ and $Q<0$, then $C$ has a real double root $y_{1}$ and a simple real root $y_{2}$ and $y_{1}<y_{2}$,

- if $D=0$ and $Q>0$, then $C$ has a real double root $y_{1}$ and a simple real root $y_{2}$ and $y_{1}>y_{2}$,

- if $D=0$ and $Q=0$, then $P=0$ and $C$ has a real triple root.

Remark : under the change of variable $Y \mapsto-Y$, the numbers $P$ and $D$ are invariant and the number $Q$ turns into its opposite. 


\subsection{Encoding the topology of a real algebraic trigonal curve}

Let us now consider a $\mathcal{L}$-nonsingular real algebraic trigonal curve $C$ in $\Sigma_{n}$. Let us choose a standard coordinate system $(x, y)$ on $\Sigma_{n}$ such that the fiber at infinity intersects $C$ in 3 distinct points (not necessarily real). The equation of $C$ in $\mathbb{C}^{2}=\Sigma_{n} \backslash\left(E \cup F_{\infty}\right)$ is

$$
C(X, Y)=Y^{3}+a_{1}(X) Y^{2}+a_{2}(X) Y+a_{3}(X)
$$

where $a_{j}(X)$ is a polynomial of degree $j n$.

As in section 4.1, define the three following polynomial in one variable

$$
P(X)=a_{2}(X)-\frac{a_{1}(X)^{2}}{3}, \quad Q(X)=a_{3}(X)-\frac{a_{2}(X) a_{1}(X)}{3}+\frac{2 a_{1}(X)^{3}}{27}, \quad D(X)=-4 P(X)^{3}-27 Q(X)^{2} .
$$

The curve $C$ is $\mathcal{L}$-nonsingular so $D(X)$ has only simple roots. Denote by $x_{1}<x_{2}<\ldots<x_{l}$ the roots of $D(X)$. Denote also by $s_{0}$ the sign of $D(x)$ with $x<x_{1}$ and $s_{i}$ the sign of $Q\left(x_{i}\right)$ for $i \in\{1 \ldots l\}$.

Definition 4.1 The sign array $\left[s_{0}, s_{1} \ldots s_{l}\right]$ is called the sign array of the curve $C$ in the chosen standard coordinate system.

From the definition one can see that the sign array determines the position of a $\mathcal{L}$-nonsingular real algebraic trigonal curve in $\Sigma_{n}$ with respect to $\mathcal{L}$ and conversely provided that a standard coordinate system has been chosen.

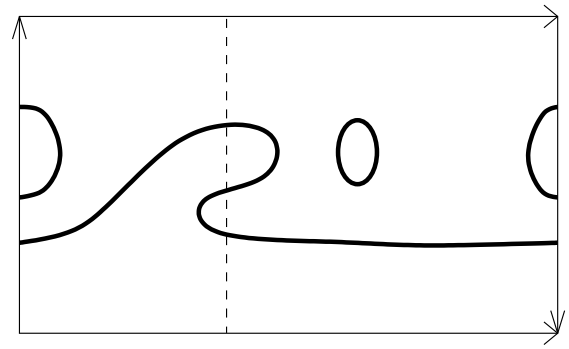

a)

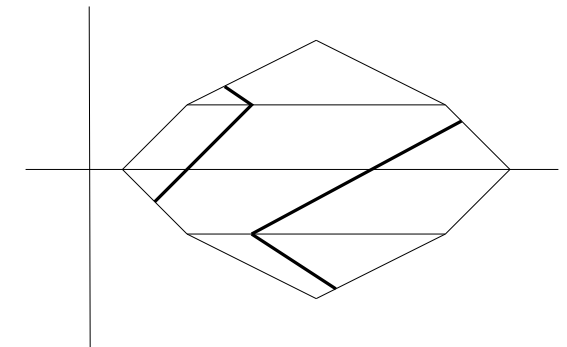

b)

Figure 1:

Example : Consider the trigonal curve on $\Sigma_{1}$ depicted in Figure 17) and suppose that a standard coordinate system is chosen such that the vertical edges of the rectangle represent the fiber at infinity. Then the sign array of this trigonal curve is $[+,+-++++]$.

\section{An order on the polygons of a subdivision}

Let $\Delta$ be the triangle with vertices $(0,0),(0, d)$ and $(n d, 0)$ and let $\tau$ be a subdivision of $\Delta$ into convex integer polygons. We give an algorithm to put an order on the polygons of $\tau$. In the Viro method, it will correspond to the order in which the polygons are scanned by the pencil of lines. Roughly speaking, the polygons will be scanned from the left to the right and only one polygon will be scanned at the same time except if it contains a horizontal edge. If two polygons have a horizontal edge in common, they will be scanned simultaneously by the pencil. 


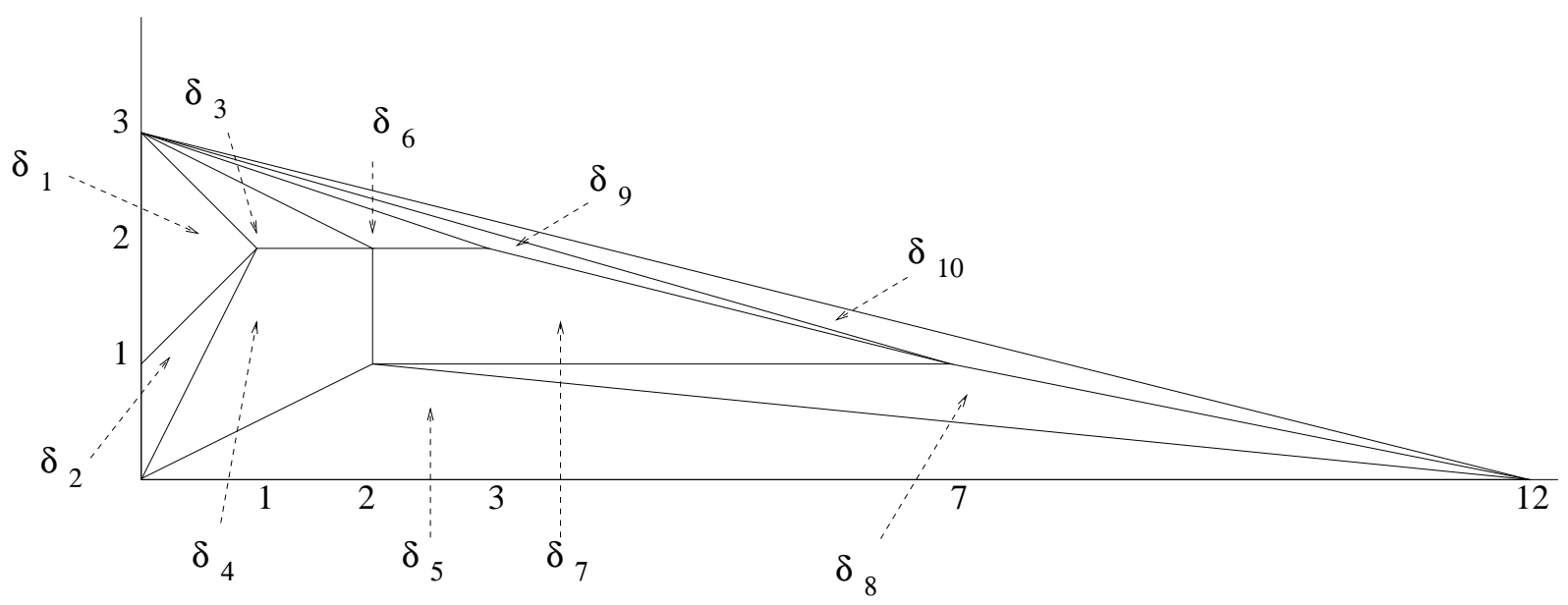

Figure 2: Order on polygons

In the procedure, $\theta$ is the current ordered subset of the 2-dimensional cells of $\tau$, and $\xi$ is the set of remaining cells. The procedure starts with $\theta=()$ and $\xi=\{2$-dimensional cells of $\tau\}$. Perform the following operations until $\xi=\emptyset$.

Step 1 : Suppose that $\theta=\left(\Phi_{1}, \ldots, \Phi_{j-1}\right)$ and put

- $U=\bigcup_{\delta \in \xi} \delta$,

- $\Lambda=\left\{(x, y) \mid x=\min _{(z, y) \in U}(z)\right\}$

(i.e. $\Lambda$ is the left side of $U$ ),

- $\widetilde{\xi}=\{\delta \in \xi \mid \delta$ has an edge which is contained in $\Lambda\}$

(i.e. elements of $\widetilde{\xi}$ are the leftest polygons of $\xi$ ),

- $\widetilde{\Lambda}=\left\{(\delta,(x, y)) \in \widetilde{\xi} \times \Lambda \mid(x, y) \in \delta, y=\max _{(z, w) \in \delta}(w)\right.$ and $\delta$ has no horizontal edge containing $(x, y)\}$

(i.e. we take pairs $(\delta,(x, y))$ made of a polygon $\delta$ and a distinguished vertex $(x, y)$ of $\delta$ such that $(x, y)$ is the higher point of $\delta$ and is not on one of its horizontal edge),

- $\left\{\left(\delta_{0},\left(x_{0}, y_{0}\right)\right)\right\}=\left\{(\delta,(x, y)) \in \widetilde{\Lambda} \mid y=\min _{\left(\delta^{\prime},(z, w)\right) \in \widetilde{\Lambda}}(w)\right\}$

(i.e. we take the element of $\widetilde{\Lambda}$ with the distinguished vertex of smaller ordinate).

Step 2 : Here we look for all the polygons which will be scanned at the same time by the pencil of lines.

Define by induction some polygons $\left(\delta_{i}\right)_{0<i<k}$, where $\delta_{0}$ has been defined at step 1 : if $\delta_{l}$ shares a horizontal edge with a polygon $\delta^{\prime}$, distinct from $\delta_{l-1}$ if $l>0$, then put $\delta_{l+1}=\delta^{\prime}$. Else, put $k=l$ and $\Phi_{j}=\left\{\delta_{i}, 0 \leq i \leq k\right\}$.

Step 3: Put $\xi=\xi \backslash \Phi_{j}$. If $x_{0}>1$ then also put $\theta=\left(\Phi_{1}, \ldots, \Phi_{j}\right)$.

Remark : in this algorithm, we "throw away" polygons corresponding to curves of degree 1 in $Y$ and which do not have a horizontal edge of height 1 .

Example : Applying this algorithm to the (non-convex) subdivision shown in Figure 2, one obtains

$$
\theta=\left(\left\{\delta_{1}\right\},\left\{\delta_{2}\right\},\left\{\delta_{3}, \delta_{4}\right\},\left\{\delta_{6}, \delta_{7}, \delta_{8}\right\},\left\{\delta_{9}\right\},\left\{\delta_{10}\right\}\right) .
$$




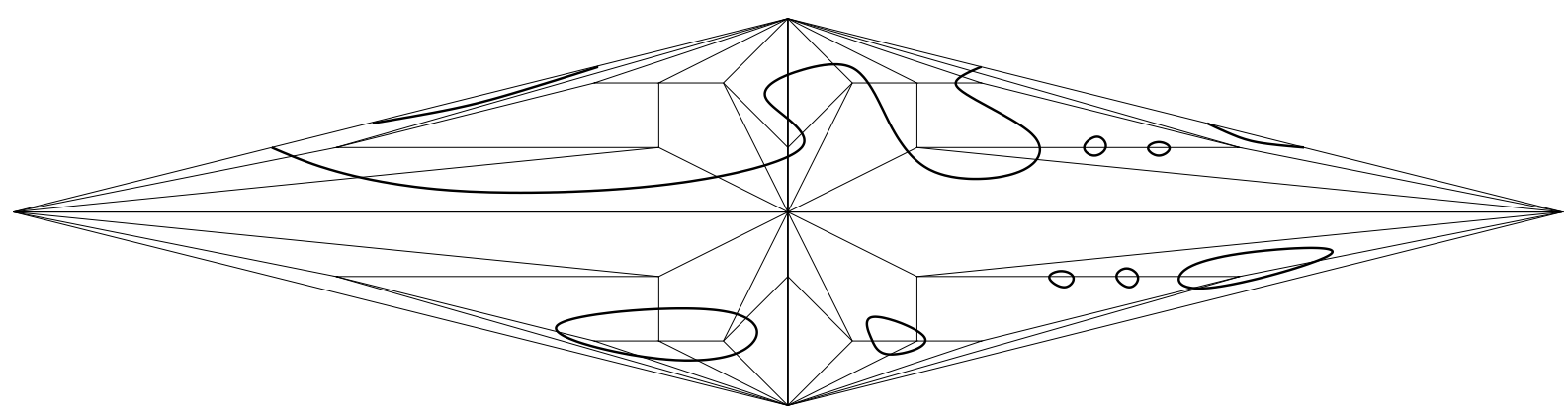

Figure 3:

Let $\Phi$ be an element of $\theta$ and $\delta$ a polygon of $\Phi$.

Definition 5.1 The height of $\delta$ is defined as $\max _{(x, y) \in \delta}(y)$. The height of $\Phi$ is the maximum of the heights of the polygons of $\Phi$.

Note that by construction, all the elements of $\Phi$ have height at least 2 .

\section{The sign array associated to a trigonal patchwork}

To a trigonal patchwork, we associate a sign array encoding the position that the curve we want to construct would have with respect to $\mathcal{L}$. This array is constructed studying where should be located the roots of the discriminant of the desired curve, and what should be the sign of $Q(X)$ at those points.

Let $\Pi$ be a trigonal patchwork satisfying conditions (1) and (2) of section 3 Following what happens when the subdivision is convex (see $[\underline{\mathrm{LdM}}]$ ), the roots $x_{0} \in \mathbb{R}$ of $D(X)$ will be obtained out of $\Pi$ in exactly three different ways:

- any root in $\mathbb{R}^{*}$ of the discriminant a curve $C_{i}$ of the patchwork gives rise to such a root $x_{0}$,

- suppose there is a horizontal edge of height 1 contained in a polygon $\delta_{1}$ of height at least 2 and in a polygon $\delta_{2}$ of height 1 . The polygon $\delta_{1}$ corresponds in the patchwork to a curve of equation $\alpha Y^{3}+a_{1}(x) Y^{2}+a_{2}(X) Y$ and the polygon $\delta_{2}$ corresponds to a curve of equation $a_{2}(X) Y+a_{3}(X)$.

Then a root of $x_{1} \in \mathbb{R}^{*}$ of $a_{2}(X)$ such that $a_{1}\left(x_{1}\right)$ and $a_{3}\left(x_{1}\right)$ have the same sign (see Figure 1b)) gives rise to two such roots $x_{0}$.

- suppose there is a horizontal edge of height 2 contained in a polygon $\delta_{1}$ of height 3 and in a polygon $\delta_{2}$ of height 2. The polygon $\delta_{1}$ corresponds in the patchwork to a curve of equation $Y^{3}+a_{1}(x) Y^{2}$ and the polygon $\delta_{2}$ corresponds to a curve of equation $a_{1}(X) Y^{2}+a_{2}(X) Y+a_{3}(X)$.

Then a root $x_{1} \in \mathbb{R}^{*}$ of $a_{1}(X)$ such that $a_{2}\left(x_{1}\right)$ is positive (see Figure 4 )) gives rise to two such roots $x_{0}$.

First, we associate two lists of signs to each element of $\theta$.

\subsection{Lists of signs associated to an element of $\theta$}

Let $\Phi_{i}$ be the $i^{\text {th }}$ element of $\theta$ and let be $a_{1}(X), a_{2}(X)$ and $a_{3}(X)$ all the polynomials that occur in the polygons of $\Phi_{i}$. The polynomial $a_{j}(X)$ is the coefficient of $Y^{(3-j)}$ for some polygon of $\Phi_{i}$. 


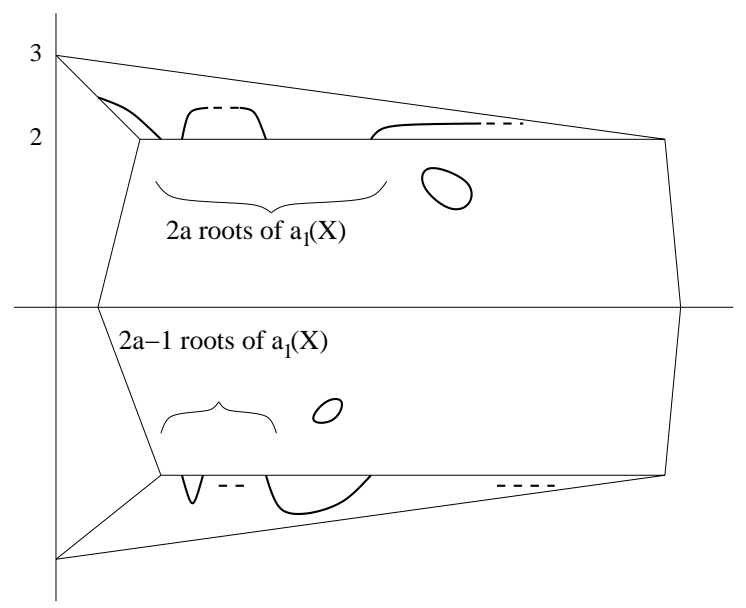

a)

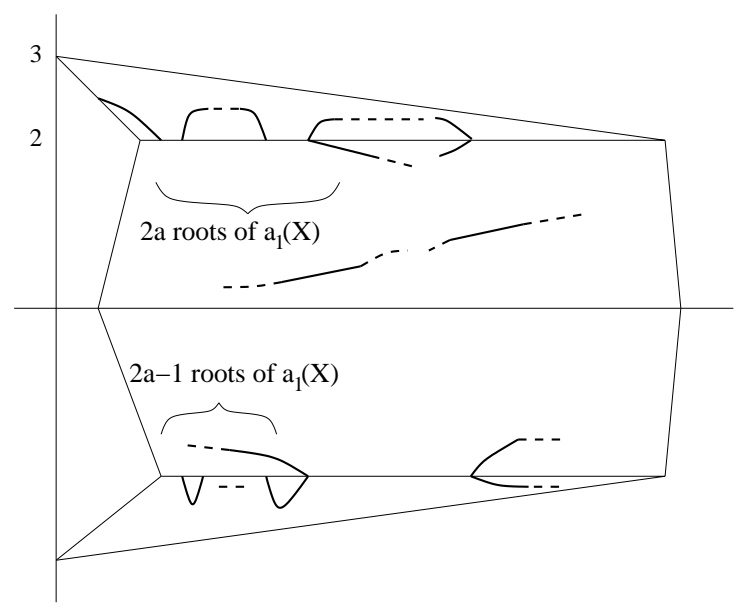

b)

Figure 4:

We define $x_{i, 1}^{-}<\ldots<x_{i, u_{i}}^{-}\left(\right.$resp. $\left.x_{i, 1}^{+}<\ldots<x_{i, v_{i}}^{+}\right)$to be all the numbers in $\mathbb{R}_{-}^{*}$ (resp. $\mathbb{R}_{+}^{*}$ ) which verify one of the following conditions

- $x_{i, l}^{s}$ is a root of the discriminant of the curve corresponding to one of the polygons of $\Phi_{i}$,

- two polygons of $\Phi_{i}$ have a common horizontal edge of height 1 , and $x_{i, l}^{s}$ is a root of $a_{2}(X)$ such that $a_{1}\left(x_{i, l}^{s}\right)$ and $a_{3}\left(x_{i, l}^{s}\right)$ have the same sign (see Figure [b)).

- two polygons of $\Phi_{i}$ have a common horizontal edge of height 2 , and $x_{i, l}^{s}$ is a root of $a_{1}(X)$ such that $a_{2}\left(x_{i, l}^{s}\right)$ is positive (see Figure 4).

\subsection{1 $\Phi_{i}$ is of height 3 and does not contain any polygon of height 2}

That means that $\Phi_{i}$ contains a polygon corresponding to a curve of degree 3 and that no one of the $x_{i, j}^{s}$ comes from an edge of height 2 . Denote by $\sigma_{i}^{s}$ the sign of $Q_{i}\left(x_{i, l}^{s}\right)$ and perform the following substitutions in the lists $x_{i, 1}^{-} x_{i, 2}^{-} \ldots x_{i, u_{i}}^{-}$and $x_{i, 1}^{+} x_{i, 2}^{+} \ldots x_{i, u_{i}}^{+}$

- if $x_{i, l}^{s}$ is a root of the discriminant of the curve of degree 3 , then replace $x_{i, l}^{s}$ by $\sigma_{l}^{s}$,

- if $x_{i, l}^{s}$ is a root of $a_{2}(X)$, then replace $x_{i, l}^{s}$ by $\sigma_{l}^{s} \sigma_{l}^{s}$ (see Figure 10$)$ ).

Example : The two lists of signs associated to $\left\{\delta_{1}\right\}$ in the patchwork depicted in Figure 3 are -+ and the empty list.

\subsection{2 $\Phi_{i}$ contains a polygon of height 2}

That means that $\Phi_{i}$ contains a polygon corresponding to a curve of degree 2. Denote by $\sigma_{i}^{s}$ the sign of $a_{1}(x)$ for $x \in \mathbb{R}_{s}^{*}$ small enough, and by $a_{l}^{s}$ the number of roots of $a_{1}(X)$ counted with multiplicity which are strictly between 0 and $x_{i, l}^{s}$. Define $\sigma_{l}^{\prime s}=(-1)^{a_{l}^{s}} \sigma_{i}^{s}$ and $\sigma_{l}^{\prime \prime s}=-\sigma_{l}^{\prime s}$ and perform the following substitutions in the lists $x_{i, 1}^{-} x_{i, 2}^{-} \ldots x_{i, u_{i}}^{-}$and $x_{i, 1}^{+} x_{i, 2}^{+} \ldots x_{i, u_{i}}^{+}$

- if $x_{i, l}^{s}$ is a root of the discriminant of the curve of degree 2 , then replace $x_{i, l}^{s}$ by $\sigma_{l}^{\prime s}$ (see Figure 田) 
- if $x_{i, l}^{s}$ is a root of $a_{2}(X)$, then replace $x_{i, l}^{s}$ by $\sigma_{l}^{\prime s} \sigma_{l}^{\prime s}$,

- if $x_{i, l}^{s}$ is a root of $a_{1}(X)$, then replace $x_{i, l}^{s}$ by $\sigma_{l}^{\prime \prime s} \sigma_{l}^{\prime s}$ (see Figure 4b)).

Example : The two lists of signs associated to $\left\{\delta_{6}, \delta_{7}, \delta_{8}\right\}$ in the patchwork depicted in Figure 3 are the empty list and -+---------

\subsection{The final sign array}

So for each element $\Phi_{i}$ of $\tau$, we have defined two lists of signs $\sigma_{i, 1}^{-} \ldots \sigma_{i, u_{i}^{\prime}}^{-}$and $\sigma_{i, 1}^{+} \ldots \sigma_{i, v_{i}^{\prime}}^{+}$.

Define $b_{(3-i) n, i}$ to be the coefficient of the monomial $X^{(3-i) n} Y^{i}$ in the patchwork and put $\widetilde{C}(Y)=$ $\sum_{i=0}^{3} b_{(3-i) n, i} Y^{i}$. Denote by $s_{0}$ the sign of the discriminant of $\widetilde{C}(Y)$.

Definition 6.1 The sign array associated to the patchwork is

$$
\left[s_{0}, \sigma_{k, 1}^{-} \sigma_{k, 2}^{-} \ldots \sigma_{k, u_{k}^{\prime}}^{-} \sigma_{k-1,1}^{-} \ldots \sigma_{k-1, u_{k-1}^{\prime}}^{-} \ldots \ldots \sigma_{1,1}^{-} \ldots \sigma_{1, u_{1}^{\prime}}^{-} \sigma_{1,1}^{+} \ldots \sigma_{1, v_{1}^{\prime}}^{+} \sigma_{2,1}^{+} \ldots \sigma_{2, v_{2}^{\prime}}^{+} \ldots \ldots \sigma_{k, 1}^{+} \ldots \sigma_{k, v_{k}^{\prime}}^{+}\right]
$$

Example : The sign array associated to the trigonal patchwork depicted in Figure 3 is

$$
[+,+--+---+------------]
$$

We can now state our main theorem whose proof will be given in section 10 Proposition 10.4

Theorem 6.2 Let $\Pi$ be a trigonal patchwork of degree $n$ which satisfy the hypothesis (1) and (2) of section 3 . Then there exists a $\mathcal{L}$-nonsingular real algebraic trigonal curve in $\Sigma_{n}$ realizing the sign array associated to $\Pi$.

Using Theorem 6.2, we can now prove Theorem 3.4.

Proof of Theorem 3.4: If the hypothesis (1) and (2) of section 3 are fulfilled, then Theorem 3.4 is a consequence of Theorem 6.2. Indeed, any $\mathcal{L}$-nonsingular real algebraic trigonal curve realizing the sign array associated to $\Pi$ has a chart which is isotopic to the union of the charts used in the patchwork.

If only the hypothesis (1) is fulfilled, one can perturb slightly the coefficients of the curves used in the patchwork in order that the hypothesis (2) of section 3 is fulfilled. Then apply Theorem 6.2 to this patchwork. Perturbing in different ways the initial patchwork, one can construct $\mathcal{L}$-nonsingular real algebraic trigonal curve realizing different positions with respect to the pencil $\mathcal{L}$. However, all these curves have isotopic charts and this isotopy type is the one required by Theorem 3.4

\section{Signed real rational graphs associated to trigonal curves and to curves of bidegree $(2, n)$}

\subsection{Signed real rational graphs}

Here we recall some fact about real rational graphs. We refer to [Bru06], Bru], Ore03] and [NSV02] for more details and proofs.

Color and orient $\mathbb{R} P^{1}$ as depicted in Figure 5 ). 


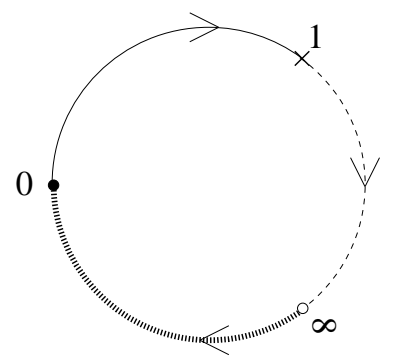

a)

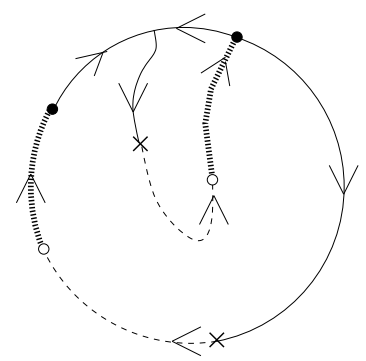

b)

Figure 5:

Definition 7.1 Let $\Gamma$ be a graph on $\mathbb{C} P^{1}$ invariant under the action of the complex conjugation and $\pi: \Gamma \rightarrow \mathbb{R} P^{1}$ a continuous map. Then the coloring and orientation of $\mathbb{R} P^{1}$ shown in Figure 5 a) defines a coloring and an orientation of $\Gamma$ via $\pi$.

The graph $\Gamma$ equipped with this coloring and this orientation is called a real rational graph if

- any vertex of $\Gamma$ has an even valence,

- for any connected component $W$ of $\mathbb{C} P^{1} \backslash \Gamma$, the map $\pi_{\mid \partial W}$ is a covering of $\mathbb{R} P^{1}$ of degree $d_{W}$,

- for any connected component $W$ of $\mathbb{C} P^{1} \backslash \Gamma$, the orientation induced by $\pi$ on $\partial W$ is also induced by an orientation of $W$.

The sum of the degrees $d_{W}$ for all connected component $W$ of $\{\operatorname{Im}(z)>0\} \backslash \Gamma$ of is called the degree of $\Gamma$.

Since a real rational graph is invariant under the complex conjugation, we will draw in this article only one of the two halves of real rational graphs. This half will be drawn in a disk and the boundary of this disk will be $\mathbb{R} P^{1}$.

Let $f: \mathbb{C} P^{1} \rightarrow \mathbb{C} P^{1}$ be a real rational map of degree $d$, and let $\Gamma$ be $f^{-1}\left(\mathbb{R} P^{1}\right)$ with the coloring and the orientation induced by those chosen on $\mathbb{R} P^{1}$. Then $\Gamma$ is a real rational graph of degree $d$.

Definition 7.2 One says that $\Gamma$ is the real rational graph associated to $f$.

Example : the real rational graph associated to the real rational map $f(X, Z)=\frac{(X-Z)(X-2 Z)^{2}}{(X+3 Z)\left(X^{2}+X Z+Z^{2}\right)}$ is depicted in Figure $5 \mathrm{~b}$ ).

The next theorem shows that the converse is also true : one can construct real rational map out of a real rational graph.

Theorem 7.3 Let $\Gamma$ be a real rational graph of degree $d$. Then there exists a real rational map of degree $d$ whose real rational graph is equivariantly isotopic to $\Gamma$.

Such a real rational map is said to realize $\Gamma$.

One of the steps in the proof of our main Theorem is the gluing of some real rational graphs. These gluings will not depend only on the real rational graphs, but also on some signs of polynomials which define it and on some coordinate system on $\mathbb{C} P^{1}$. This motivates the notions of marked and signed real rational graphs. 
Definition 7.4 A marked real rational graph is a real rational graph on $\mathbb{C} P^{1}$ where some coordinate system is fixed. In particular, the points 0 and $\infty$ are prescribed on such a graph.

Let $\Gamma$ be a marked real rational graph and $f$ a real rational map which realizes $\Gamma$. The marked graph $\Gamma$ is called a signed real rational graph if each connected component of $\left(\Gamma \cap \mathbb{R} P^{1}\right) \backslash\left(f^{-1}(\{0,1, \infty\}) \cup\right.$ $\{0, \infty\})$ is enhanced with a pair of signs.

As explained above, we will represent a real rational graph by a graph in a disk lying in $\mathbb{R}^{2}$. A marked real rational graph will be represented in a disk which is symmetric with respect to the $x$-axis. If $\left(x_{1}, 0\right)$ and $\left(x_{2}, 0\right)$ are the two intersection points of $\{y=0\}$ and the circle bounding this disk with $x_{1}<x_{2}$, then $\left(x_{1}, 0\right)$ (resp. $\left.\left(x_{2}, 0\right)\right)$ will be 0 (resp. $\infty$ ) on the marked graph. The positive (resp. negative) points of $\mathbb{R} P^{1}$ will be drawn on the half plane $\{(x, y) \mid y>0\}$ (resp. $\left.\{(x, y) \mid y<0\}\right)$ and is called the positive (resp. negative) part of the graph.

In the rest of this paper, we will not draw the arrows on the real rational graphs.

Example : A signed real rational graph is depicted on figure 6b).

\subsection{Signed real rational graph associated to a real algebraic trigonal curve in $\Sigma_{n}$}

Let $C$ be a real algebraic trigonal curve in $\Sigma_{n}$. Then $C$ has the following equation in $\Sigma_{n} \backslash E$

$$
C(X, Y, Z)=Y^{3}+a_{1}(X, Z) Y^{2}+a_{2}(X, Z) Y+a_{3}(X, Z)
$$

where $a_{j}(X, Z)$ is a homogeneous polynomial of degree $j n$. To such a curve, we associate the real rational graph $\Gamma(C)$ given by the map

$$
\begin{array}{cccc}
f: \mathbb{C} P^{1} & \longrightarrow & \mathbb{C} P^{1} \\
& {[X: Z]} & \longmapsto & \frac{-4 P(X, Z)^{3}}{27 Q(X, Z)^{2}}
\end{array}
$$

where the polynomials $P$ and $Q$ are those defined in section 4.2

Definition 7.5 The graph $\Gamma(C)$ is called the real rational graph associated to $C$.

The preimages of 1 by $f$ correspond to the roots of $D(X, Z)$, the discriminant of $C$. So if the curve $C$ is $\mathcal{L}$-nonsingular, according to section 4 one can recover the position of $C$ in $\Sigma_{n}$ with respect to $\mathcal{L}$ up to the transformation $Y \mapsto-Y$ only from $\Gamma \cap \mathbb{R} P^{1}$.

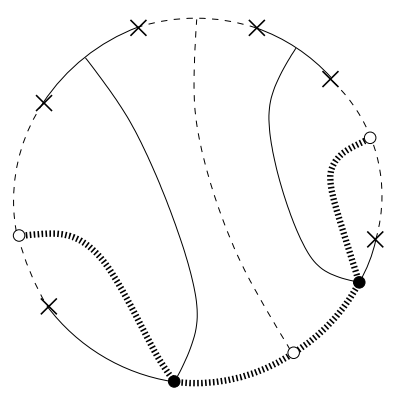

a)

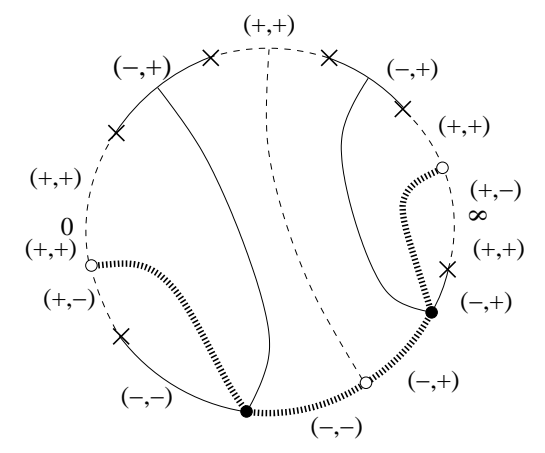

b)

Figure 6: 
Example : the real rational graph associated to the trigonal curve in $\Sigma_{1}$ depicted on Figure $1 \mathrm{a}$ ) is depicted in Figure 6a).

Conversely, suppose that using Theorem [7.3. we have constructed a real rational map $f(X, Z)=$ $\frac{-4 P(X, Z)^{3}}{27 Q(X, Z)^{2}}$ out of a real rational graph $\Gamma$. Then, $\Gamma$ is the real rational graph associated to the trigonal curve $Y^{3}+P(X, Z) Y+Q(X, Z)$.

Up to rotation, this real rational graph does not depend on the coordinate system we choose on $\Sigma_{n} \backslash E$. If now we take into account the coordinate system, we obtain a marked real rational graph. Let us turn it into a signed real rational graph $\Gamma_{ \pm}(C)$ : the pair of signs on each connected component of $\left(\Gamma \cap \mathbb{R} P^{1}\right) \backslash\left(f^{-1}(\{0,1, \infty\}) \cup\{0, \infty\}\right)$ is the pair formed by the sign of $D(X)$ and the sign of $Q(X)$ on this component.

Definition 7.6 The graph $\Gamma_{ \pm}(C)$ is called the signed real rational graph associated to the polynomial $C$.

Example : Suppose that we have fixed the coordinate system in $\Sigma_{1}$ such that the vertical edges of the rectangle in Figure 10 ) are the fiber at infinity and the fiber in dotted line is the fiber at 0. Then $\Gamma_{ \pm}(C)$ is depicted in Figure 6b).

Note that the signed real rational graph associated to a trigonal curve in some coordinate system can be extracted from the knowledge of the real rational graph associated to this curve and the topology of the curve. For this reason, we won't write explicitly the pairs of signs on signed real rational graph associated to trigonal curves in the rest of this paper.

Here we give a list of necessary and sufficient conditions for a signed real rational graph to be associated to some $\mathcal{L}$-nonsingular real algebraic trigonal curve. The proof can be found in Ore03.

Proposition 7.7 Let $\Gamma_{ \pm}$be a signed real rational graph and let $\pi: \Gamma_{ \pm} \rightarrow \mathbb{R} P^{1}$ be a continuous map as in definition 7.1. Then there exists a $\mathcal{L}$-nonsingular real algebraic trigonal curve in $\Sigma_{n}$ such that $\Gamma_{ \pm}(C)$ is equivariantly isotopic to $\Gamma_{ \pm}$if and only if the following conditions are fulfilled

- $\Gamma_{ \pm}$is of degree $6 n$,

- any preimage of 0 has an order which is divisible by 3 ,

- any preimage of $\infty$ has an order which is divisible by 2 ,

- any preimage of 1 is of order 1 ,

- any pair of sign $(+, s)$ labels a connected component of $\pi^{-1}(] \infty, 0[)$ or $\pi^{-1}(] \infty, 0[)$,

- any pair of sign $(-, s)$ labels a connected component of $\pi^{-1}(] 1, \infty[)$,

- when passing through a preimage of $\infty$ of order $2 a$, the pair of sign $\left(s^{\prime}, s\right)$ becomes $\left(s^{\prime},(-1)^{a} s\right)$. 


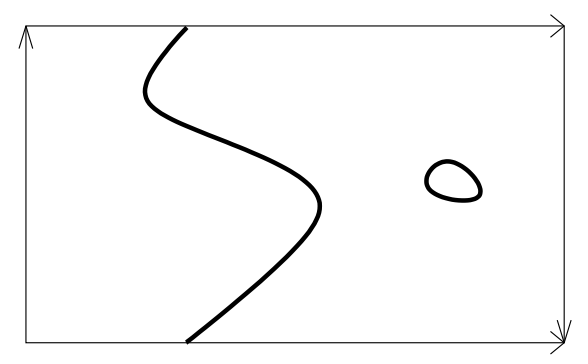

a)

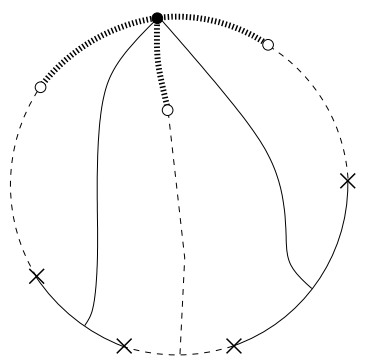

b)

Figure 7:

\subsection{Signed real rational graph associated to a real algebraic curve of bidegree $(2, \mathrm{n})$ in $\Sigma_{n}$}

Let $C$ be a real algebraic curve of bidegree $(2, n)$ in $\Sigma_{n}$. So $C$ has the following equation in $\Sigma_{n} \backslash E$

$$
C(X, Y, Z)=a_{1}(X, Z) Y^{2}+a_{2}(X, Z) Y+a_{3}(X, Z)
$$

where $a_{j}(X, Z)$ is a homogeneous polynomial of degree $j n$. The intersections of the curve $C(X, Y, Z)$ with the exceptional divisor correspond to the roots of $a_{1}(X, Z)$. To such a curve, we associate the real rational graph $\Gamma(C)$ associated to the map

$$
\begin{array}{cccc}
f \quad: & \mathbb{C} P^{1} & \longrightarrow & \mathbb{C} P^{1} \\
& {[X: Z]} & \longmapsto & \frac{-a_{1}(X, Z)^{4}}{a_{2}(X, Z)^{2}-4 a_{1}(X, Z) a_{3}(X, Z)-a_{1}(X, Z)^{4}}
\end{array} .
$$

Definition 7.8 The graph $\Gamma(C)$ is called the real rational graph associated to $C$.

The real rational graph associated to the curve $C$ appears as a limit of the real rational graph associated to a real trigonal algebraic curve when its coefficient of $Y^{3}$ tends to 0 .

The preimages of 1 by $f$ correspond to the roots of $a_{2}(X, Z)^{2}-4 a_{1}(X, Z) a_{3}(X, Z)$, the discriminant of $C$.

Example : the real rational graph associated to the curve of bidegree $(1,2)$ in $\Sigma_{1}$ depicted in Figure 73) is depicted in Figure [7b).

As in the case of trigonal curves, the real rational graph associated to $C$ does not depend on the coordinate system we choose on $\Sigma_{n} \backslash E$, up to rotation. Taking into account the coordinate system chosen to write the equation of $C$, we obtain a marked real rational graph. Let us turn it into a signed real rational graph $\Gamma_{ \pm}(C)$ : the pair of signs on each connected component of $\left(\Gamma \cap \mathbb{R} P^{1}\right) \backslash\left(f^{-1}(\{0,1, \infty\}) \cup\{0, \infty\}\right)$ is the pair formed by the sign of $a_{2}(X, Z)^{2}-4 a_{1}(X, Z) a_{3}(X, Z)$ and the sign of $a_{1}(X)$ on this component.

Definition 7.9 The graph $\Gamma_{ \pm}(C)$ is called the signed real rational graph associated to the polynomial $C$.

This definition of $\Gamma_{ \pm}$for a curve of bidegree $(2, n)$ is motivated by the fact that when we will glue together the curves of the patchwork in section 9 the topology coming from a curve of degree 2 will be encoded by the sign of $a_{1}(X)$ (see Figure 4 ). 
Note that the knowledge of the real rational graph and the topology of $C$ does not allow one to recover the sign real rational graph. Indeed, the sign of $a_{1}(X)$ can be recovered neither from the real rational graph nor from the topology of the curve.

However, when the curve $C$ will be used in a patchwork, the sign of some other polynomials will allow us to recover the sign of $a_{1}(X)$.

\section{Associating a signed real rational graph to any element of $\theta$}

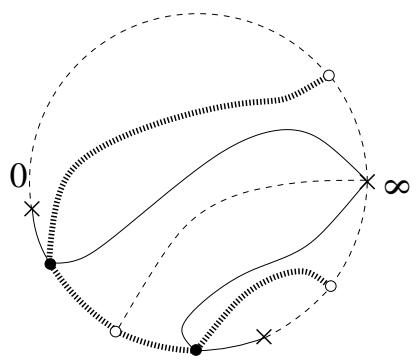

a) $\left\{\delta_{1}\right\}$

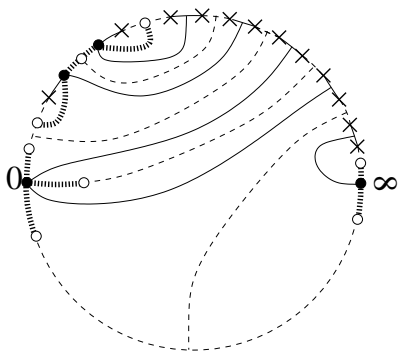

a) $\left\{\delta_{6}, \delta_{7}, \delta_{8}\right\}$

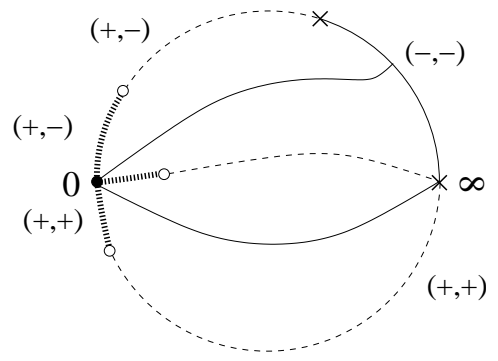

b) $\left\{\delta_{2}\right\}$

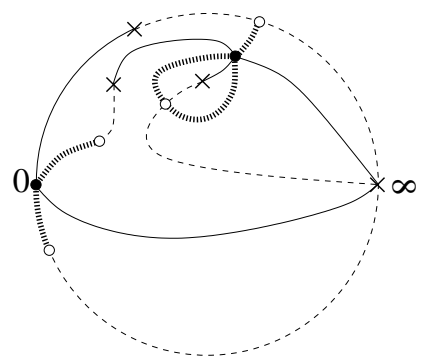

c) $\left\{\delta_{3}, \delta_{4}\right\}$

Figure 8:

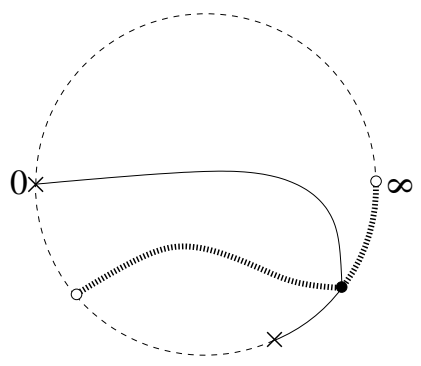

b) $\left\{\delta_{9}\right\}$

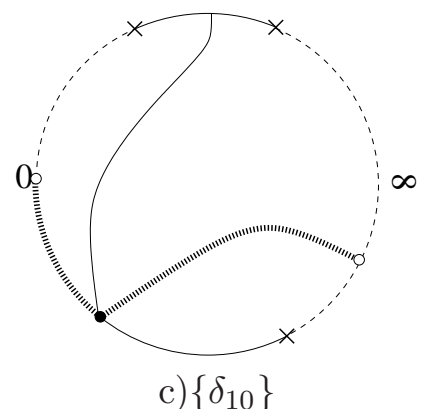

c) $\left\{\delta_{10}\right\}$

Figure 9:

Let $\Pi$ be a trigonal patchwork of degree $n$ satisfying conditions (1) and (2) of section 3 and let $\theta=\left(\Phi_{1}, \ldots, \Phi_{k}\right)$ be the $k$-tuple constructed in section [5] We are going to associate a signed real rational graph $\Gamma_{ \pm}\left(\Phi_{i}\right)$ to each $\Phi_{i}$. Dealing with charts of polynomials implies that a coordinate system is fixed. The signed real rational graphs will be constructed in this coordinate system.

\section{1 $\Phi_{i}$ is of height 2}

Here we consider signed real rational graphs of curves of bidegree $(2, n)$ in $\Sigma_{n}$. As mentioned in section [7.3. it cannot be extracted only from the knowledge of the real rational graphs of those curves and of their topology. However, when such a curve is used in a patchwork of a trigonal curve, one can recover the sign of $a_{1}(X)$ out of this patchwork.

Indeed, if $\delta$ is a polygon of height 2 in a trigonal patchwork, let $p=(x, 2)$ be the leftest point of height 2 of $\delta$ (i.e. $x=\min _{(z, 2) \in \delta}(z)$ ). Then, $[(0,3) ; p]$ is an edge of the subdivision, and any curve of degree 3 in the patchwork whose Newton polygon contains this edge prescribes the sign of $a_{1}(X)$ for small values of $X$ : if the chart of one of these cubic intersect the edge $[(0,3) ; p]$ (resp. 
$[(0,3) ;(-x, 2)])$, then $a_{1}(X)$ is negative for small positive (resp. negative) values of $X$. This is because $[(0,3) ;(-x, 2)]$ is of integer length 1 and the coefficient of $Y^{3}$ is 1 .

For this reason, we will not write explicitly the pairs of signs on signed real rational graphs associated to a curve of bidegree $(2, n)$ in a trigonal patchwork.

\subsection{1 $\Phi_{i}=\{\delta\}$}

That means that $\delta$ is the Newton polygon of a curve $C(X, Y)=a_{1}(X) Y^{2}+a_{2}(X) Y+a_{3}(X)$ in the patchwork with $a_{1}(X)$ a monomial and $a_{3}(X)$ a non zero polynomial. Consider this curve as a curve of bidegree $(2, n)$ in $\Sigma_{n}$, i.e. homogenize the curve $C$ in the following way :

$$
C(X, Y, Z)=a_{1}(X, Z) Y^{2}+a_{2}(X, Z) Y+a_{3}(X, Z)
$$

where $a_{j}(X, Z)$ is a homogeneous polynomial of degree $j n$. This curve may have singular points on the fibers $\{X=0\}$ and $\{Z=0\}$.

Consider the signed real rational graph $\Gamma_{ \pm}(C(X, Y, Z))$ constructed in section 7.3 Then perturb this graph in such a way that all the preimages of $\infty$ are simple and perform all the operations depicted in Figure 10 on $\Gamma_{ \pm}(C(X, Y, Z))$ until the signed real rational graph has no ramification point on the preimage of $\left[\infty, 0\left[\right.\right.$. The first operation occurs in $\mathbb{C} P^{1} \backslash \mathbb{R} P^{1}$ and the two last ones on $\mathbb{R} P^{1}$.

The obtained signed real rational graph is $\Gamma_{ \pm}\left(\Phi_{i}\right)$.

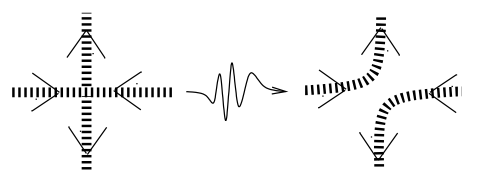

a)

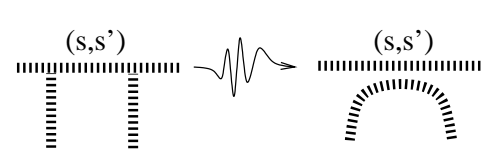

b)

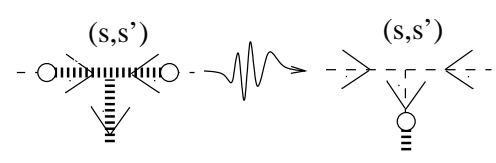

c)

Figure 10:

Example : Let us detail how to find $\Gamma_{ \pm}\left(\left\{\delta_{2}\right\}\right)$ in the patchwork depicted in Figure 3 The corresponding curve is $C_{2}(X, Y)=a X Y^{2}+b Y+c$ where $a, b$ and $c$ are real numbers. Considering $C_{2}$ as a curve in $\Sigma_{4}$, we have

$$
C_{2}(X, Y, Z)=a X Z^{3} Y^{2}+b Z^{8} Y+c Z^{12} .
$$

Then $\Gamma\left(C_{2}\right)$ is given by the real rational function

$$
f_{\delta_{2}}(x)=\frac{-a^{4} X^{4} Z^{12}}{b^{2} Z^{16}-4 a c X Z^{15}-a^{4} X^{4} Z^{12}}=\frac{-a^{4} X^{4}}{b^{2} Z^{4}-4 a c Z^{3}-a^{4} X^{4}} .
$$

Then, according to the chart of $C_{2}$, the real rational graph of $\left\{\delta_{2}\right\}$ is as depicted in Figure $\left.8 \mathrm{~b}\right)$. The chart of the curve corresponding to the polygon $\delta_{1}$ implies that $a_{1}(X)$ is negative (resp. positive) for positive (resp. negative) values of $X$. So $\Gamma_{ \pm}\left(\left\{\delta_{2}\right\}\right)$ is as depicted in Figure 8 b).

\subsection{2 $\Phi_{i}=\left\{\delta_{1}, \delta_{2}\right\}$ with $\delta_{1}$ of height 2 and $\delta_{2}$ of height 1}

Consider the signed real rational graph associated to the curve $C_{1}$ corresponding to $\delta_{1}$ as described in section 8.1.1. This curve is the union of a curve $C_{1,1}$ of degree 1 and the curve $\{Y=0\}$. So all the roots of its discriminant are double and correspond to the intersection points of $C_{1,1}$ and $\{Y=0\}$. The curve $C_{2}$ corresponding to $\delta_{2}$ tells us how to smooth these double points. More precisely, write 
$C_{1}(X, Y)=a_{1}(X) Y^{2}+a_{2}(X) Y$ and $C_{2}(X, Y)=a_{2}(X) Y+a_{3}(X)$ and suppose that $x_{0}$ is a root of $a_{2}(X)$ in $\mathbb{R}^{*}$. Then if $a_{1}\left(x_{0}\right)$ and $a_{3}\left(x_{0}\right)$ have the same sign (resp. opposite signs), then perturb the corresponding double root of the discriminant on $\Gamma_{ \pm}\left(C_{1}\right)$ into 2 distinct real roots (resp. non-real roots) as depicted in Figure 11]) (resp. 11])).

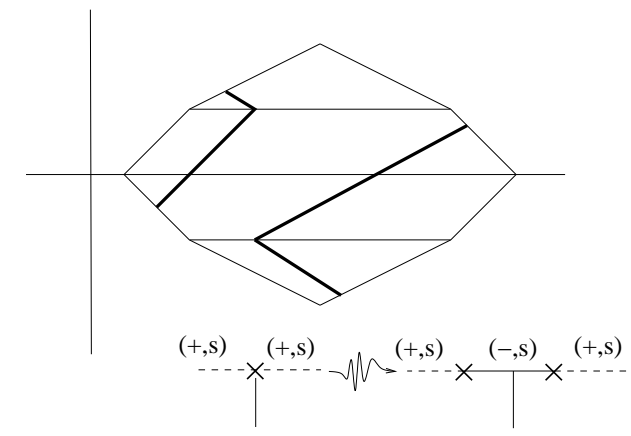

a)

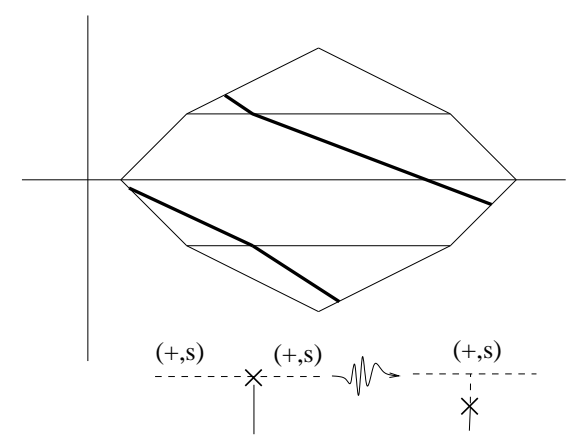

b)

Figure 11:

The graph $\Gamma_{ \pm}\left(\Phi_{i}\right)$ is the obtained signed rational graph.

Remark : The graph $\Gamma_{ \pm}\left(\Phi_{i}\right)$ is $\Gamma_{ \pm}(\widetilde{C})$, where $\widetilde{C}$ is the perturbation of $C_{1,1} \cup\{Y=0\}$ prescribed by $C_{2}$.

\section{2 $\Phi_{i}$ is of height 3}

\subsection{1 $\Phi_{i}=\{\delta\}$}

The curve $C$ in the patchwork corresponding to $\delta$ is a real algebraic trigonal curve, nonsingular in $\mathbb{C}^{*} \times \mathbb{C}$ and we define $\Gamma_{ \pm}\left(\Phi_{i}\right)$ to be $\Gamma_{ \pm}(C)$.

Example : In Figure $8 \mathrm{a})$ (resp 9b) and 98)), we have depicted $\Gamma_{ \pm}\left(\left\{\delta_{1}\right\}\right)$ (resp. $\Gamma_{ \pm}\left(\left\{\delta_{9}\right\}\right)$ and $\left.\left\{\Gamma_{ \pm}\left(\delta_{10}\right\}\right)\right)$ in the patchwork depicted in Figure 3

\subsection{2 $\Phi_{i}=\left\{\delta_{1}, \delta_{2}\right\}$ with $\delta_{1}$ of height 3 and $\delta_{2}$ of height 1}

Consider the signed real rational graph associated to the curve $C_{1}$ corresponding to $\delta_{1}$ as described in section 8.2.1 This curve is the union of a curve $C_{1,1}$ (nonsingular in $\mathbb{R}^{*} \times \mathbb{R}$ ) of degree 2 and the curve $\{Y=0\}$ and all the double roots of its discriminant correspond the intersection points of $C_{1,1}$ and $\{Y=0\}$. The curve $C_{2}$ corresponding to $\delta_{2}$ tells us how to smooth these double points. More precisely, write $C_{1}(X, Y)=Y^{3}+a_{1}(X) Y^{2}+a_{2}(X) Y$ and $C_{2}(X, Y)=a_{2}(X) Y+a_{3}(X)$ and suppose that $x_{0}$ is a root of $a_{2}(X)$ in $\mathbb{R}^{*}$. Then if $a_{1}\left(x_{0}\right)$ and $a_{3}\left(x_{0}\right)$ have the same signs (resp. opposite signs), then perturb the corresponding double root of the discriminant of the curve into 2 distinct real roots (resp. non-real roots) as in section 8.1.2

The graph $\Gamma_{ \pm}\left(\Phi_{i}\right)$ is the obtained signed rational graph.

Remark : The graph $\Gamma_{ \pm}\left(\Phi_{i}\right)$ is $\Gamma_{ \pm}(\widetilde{C})$, where $\widetilde{C}$ is the perturbation of $C_{1,1} \cup\{Y=0\}$ prescribed by $C_{2}$. In particular, $\Gamma_{ \pm}\left(\Phi_{i}\right)$ is always the signed real rational graph associated to a trigonal curve. 


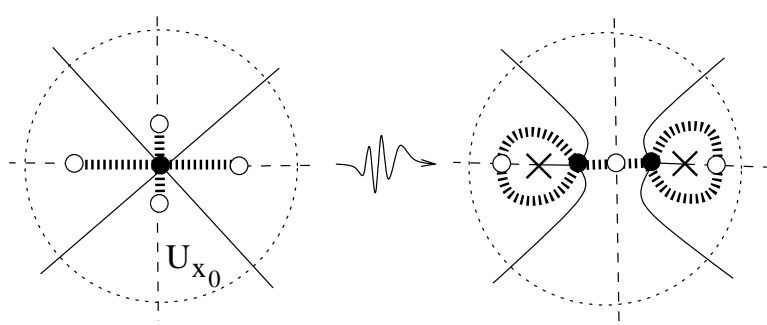

a)

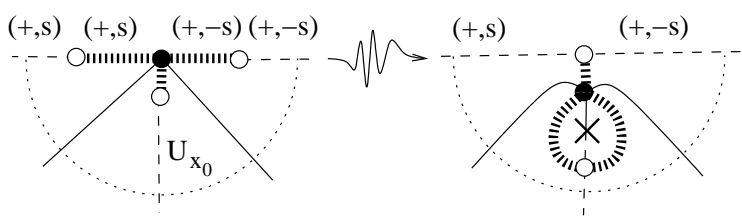

b)

Figure 12:

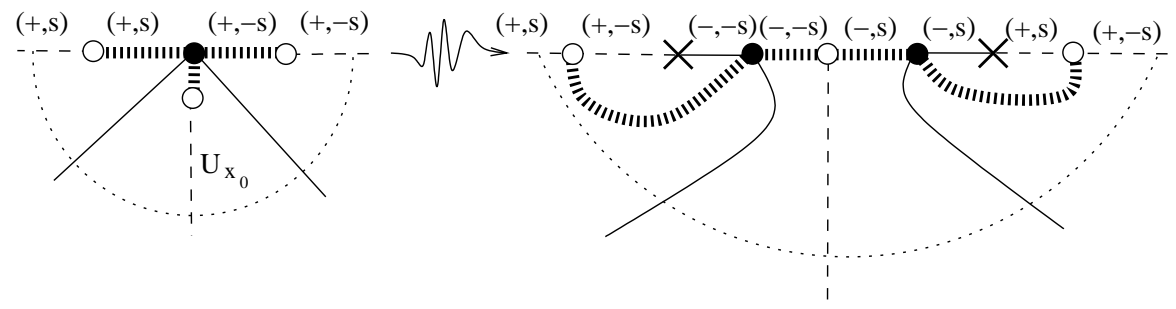

Figure 13:

\subsection{3 $\Phi_{i}=\left\{\delta_{1}, \delta_{2}\right\}$ with $\delta_{1}$ of height 3 and $\delta_{2}$ of height 2}

First, consider $\Gamma_{ \pm}\left(\delta_{2}\right)$ as explained in section 8.1.1. We will obtain $\Gamma_{ \pm}\left(\Phi_{i}\right)$ performing some modifications on $\Gamma_{ \pm}\left(\delta_{2}\right)$.

Let $x_{0}$ be a root of $a_{1}(X)$ in $\mathbb{C}^{*}$. Choose a real rational function $f$ realizing $\Gamma_{ \pm}\left(\delta_{2}\right)$ and $\epsilon>0$ such that $f$ has no ramification point on the connected component $U_{x_{0}}$ of $f^{-1}([\infty ; \epsilon])$ containing $x_{0}$. Then replace $U_{x_{0}}$ by

- the part depicted in Figure 12a) if $x_{0}$ is non real,

- the part depicted in Figure $12 \mathrm{~b})$ if $x_{0}$ is real and $a_{2}\left(x_{0}\right)<0$,

- the part depicted in Figure 13 if $x_{0}$ is real and $a_{2}\left(x_{0}\right)>0$.

The graph $\Gamma_{ \pm}\left(\Phi_{i}\right)$ is the obtained signed rational graph.

Example : In Figure 85), we have depicted $\Gamma_{ \pm}\left(\left\{\delta_{3}, \delta_{4}\right\}\right)$ in the patchwork depicted in Figure 3 .

\subsection{4 $\Phi_{i}=\left\{\delta_{1}, \delta_{2}, \delta_{3}\right\}$ with $\delta_{1}$ of height $3, \delta_{2}$ of height 2 and $\delta_{3}$ of height 1}

First, consider $\Gamma_{ \pm}\left(\delta_{2}\right)$ as explained in section 8.1.1. Then, perturb all the roots of $a_{1}(X)$ in $\mathbb{C}^{*}$ as explained in section 8.2 .3 and perturb all the double roots of the discriminant as explained in section 8.1 .2

The graph $\Gamma_{ \pm}\left(\Phi_{i}\right)$ is the obtained signed rational graph.

Example : In Figure 9 9 , we have depicted $\Gamma_{ \pm}\left(\left\{\delta_{6}, \delta_{7}, \delta_{8}\right\}\right)$ in the patchwork depicted in Figure 3

\section{Gluing of signed real rational graphs}

Let $\Pi$ be a trigonal patchwork of degree $n$ satisfying conditions (1) and (2) of section 3 and let $\theta=\left(\Phi_{1}, \ldots, \Phi_{k}\right)$ be the $k$-tuple constructed in section 5 In this section, we describe how to glue 
all the signed real rational graphs $\Gamma_{ \pm}\left(\Phi_{i}\right)$ in order to obtain a new signed real rational graph $\Gamma_{ \pm}(\Pi)$ corresponding to a nonsingular real trigonal algebraic curve in $\Sigma_{n}$.

For $i \in\{1, \ldots k-1\}$, we describe how to glue the signed real rational graph corresponding to $\Phi_{i}$ to the one corresponding to $\Phi_{i+1}$.

Example : To illustrate this procedure, we have depicted in Figure 18 all the steps in the gluing process corresponding to the patchwork depicted in Figure 3

\section{1 $\Phi_{i}$ and $\Phi_{i+1}$ are of height 3 and contain no polygon of height 2}

That means that both $\Phi_{i}$ and $\Phi_{i+1}$ contain a polygon which have the edge $\gamma=[(0,3) ;(\alpha, \beta)]$ with $\beta \leq 1$ in common.

As noticed in section 8$] \Gamma_{ \pm}\left(\Phi_{i}\right)$ and $\Gamma_{ \pm}\left(\Phi_{i+1}\right)$ correspond to two real algebraic trigonal curves, respectively $C_{i}$ and $C_{i+1}$. In particular, the two real rational graphs are given by two real rational maps

$$
f_{i}=\frac{-4 P_{i}(X, Z)^{3}}{27 Q_{i}(X, Z)^{2}}=\frac{R_{i}(X, Z)}{S_{i}(X, Z)} \text { and } f_{i+1}=\frac{-4 P_{i+1}(X, Z)^{3}}{27 Q_{i+1}(X, Z)^{2}}=\frac{R_{i+1}(X, Z)}{S_{i+1}(X, Z)} .
$$

The second form of $f_{i}$ and $f_{i+1}$ are required to be irreducible and we point out that $R_{i}(X, Z)$ can be different from $-4 P_{i}(X, Z)^{3}$.

The method here is the following : identify $\infty$ in $\Gamma_{ \pm}\left(\Phi_{i}\right)$ and 0 in $\Gamma_{ \pm}\left(\Phi_{i+1}\right)$. We obtain something which is not any more a signed real rational graph, but some kind of signed singular real rational graph. This is a graph on two copies of $\mathbb{C} P^{1}$ which are identified in one point, $\infty$ for one of them and 0 for the other one. Smoothing equivariantly this point we will obtain a smooth signed real rational graph on $\mathbb{C} P^{1}$.

\subsection{1 $\gamma=[(0,3) ;(3 l, 0)]$ or $\gamma=[(0,3) ;(2 l, 1)]$}

From condition $(1)$ we get that $f_{i}(\infty) \neq 1$ and $f_{i+1}(0) \neq 1$. Moreover, one can perturb slightly the coefficients corresponding to the integer vertices of $\gamma$ such that $f_{i}(\infty) \in \mathbb{R} P^{1} \backslash\{0,1, \infty\}$. This implies that $f_{i+1}(0) \in \mathbb{R} P^{1} \backslash\{0,1, \infty\}$.

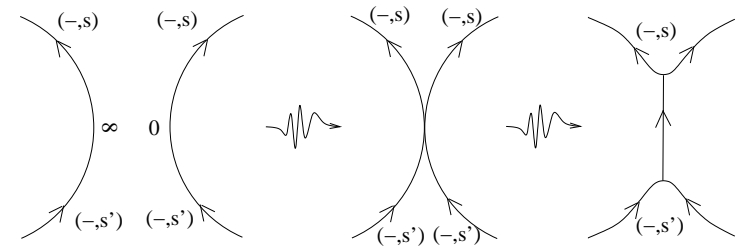

a)
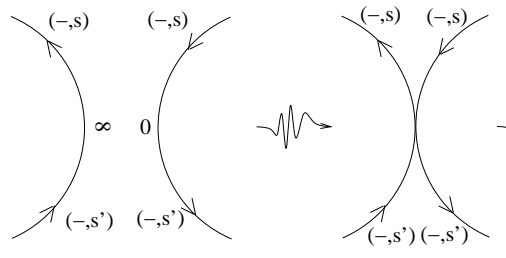

b)

Figure 14:

Then, as the truncation on $\gamma$ of $C_{i}$ and $C_{i+1}$ coincide, the pair of signs on the positive (resp. negative) part of $\Gamma_{ \pm}\left(\Phi_{i}\right)$ near $\infty$ and $\Gamma_{ \pm}\left(\Phi_{i+1}\right)$ near 0 also coincide. Suppose that $f_{i}(\infty)$ is in $] 0,1[$. Then, smooth the singular real rational graph as depicted in Figure 14a) or b), depending on the situation. The case when $f_{i}(\infty)$ is in $] 1, \infty[$ or $] \infty, 0[$ can be treated analogously.

9.1.2 $\gamma=[(0,3),(3 l-1,0)]$ or $\gamma=[(0,3),(3 l-2,0)]$

We treat the case $\gamma=[(0,3),(3 l-1,0)]$, the case $\gamma=[(0,3),(3 l-2,0)]$ being symmetric. 


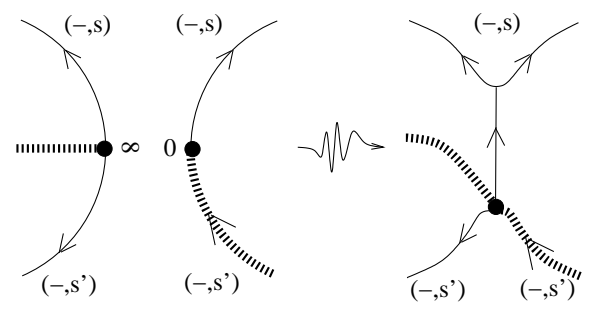

a)

Figure 15:

In this case, a simple computation shows that $\infty$ is a root of order $3 a+1$ of $R_{i}$ and that 0 is a root of order $3 b+2$ of $R_{i+1}$, where $a$ and $b$ are some natural numbers. Perturbing if necessary the coefficient of $C_{i}$ and $C_{i+1}$, one can suppose $a=b=0$. Moreover, as the truncation on $\gamma$ of $C_{i}$ and $C_{i+1}$ coincide, the pair of signs on the positive (resp. negative) part of $\Gamma_{ \pm}\left(\Phi_{i}\right)$ near $\infty$ and $\Gamma_{ \pm}\left(\Phi_{i+1}\right)$ near 0 also coincide. Then, smooth the singular real rational graph as depicted in Figure 15 or symmetrically with respect to the axis $\{Y=0\}$, depending on the situation.

\subsection{3 $\gamma=[(0,3),(2 l-1,1)]$}

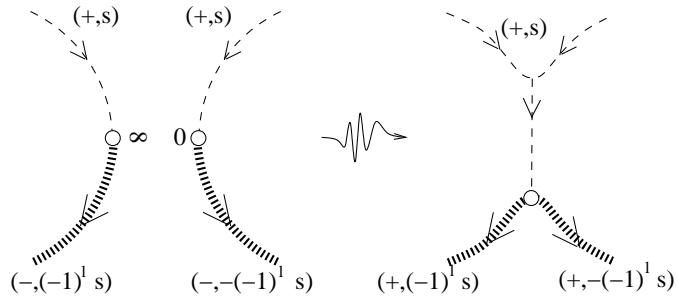

a)

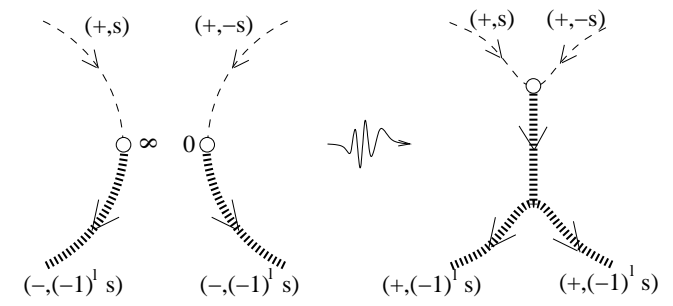

b)

Figure 16:

In this case, a simple computation shows that $\infty$ is a root of order $2 a+1$ of $S_{i}$ and that 0 is a root of order $2 b+1$ of $S_{i+1}$, where $a$ and $b$ are some natural numbers. Perturbing if necessary the coefficient of $C_{i}$ and $C_{i+1}$, one can assume that $a=b=0$. If $\left(s_{D}, s_{Q}\right)$ is the pair of signs on the positive part of $\Gamma_{ \pm}\left(\Phi_{i}\right)$ (resp. $\Gamma_{ \pm}\left(\Phi_{i+1}\right)$ ) near $\infty$ (resp. 0), then the pair of signs on the negative part of $\Gamma_{ \pm}\left(\Phi_{i}\right)$ (resp. $\left.\Gamma_{ \pm}\left(\Phi_{i+1}\right)\right)$ near $\infty$ (resp. 0) is $\left(s_{D},(-1)^{l} s_{Q}\right)$ (resp. $\left(s_{D},-(-1)^{l} s_{Q}\right)$ ). Moreover, as the truncation on $\gamma$ of $C_{i}$ and $C_{i+1}$ coincide, the sign of the discriminant on the positive (resp. negative) part of $\Gamma_{ \pm}\left(\Phi_{i}\right)$ near $\infty$ and $\Gamma_{ \pm}\left(\Phi_{i+1}\right)$ near 0 also coincide.

Then, depending on the situation, smooth the singular real rational graph using the appropriate perturbation among those depicted in Figure 16] and their symmetric with respect to the axis $\{Y=$ $0\}$.

\section{2 $\Phi_{i}$ or $\Phi_{i+1}$ contains a polygon of height 2}

Let $p=\left(x_{0}, y_{0}\right)$ be the point of the polygons of $\Phi_{i}$ of ordinate 2 with maximal abscissa. Let $\gamma$ be the unique edge of the form $[p ;(\alpha, \beta)]$ with $\beta<2$ which is a common edge of a polygon $\delta_{i}$ of $\Phi_{i}$ and of a polygon $\delta_{i+1}$ of $\Phi_{i+1}$. The line supported by $\gamma$ intersects the line $\{Y=1\}$ in the point $\left(x_{1}, 1\right)$ (see figure 17).

We suppose that $x_{1} \leq x_{0}$. The case $x_{1} \geq x_{0}$ can be recovered by symmetry. 
Let $C_{i}$ (resp. $\left.C_{i+1}\right)$ be the curve of the patchwork corresponding to $\delta_{i}\left(\operatorname{resp} . \delta_{i+1}\right)$ and $f_{i}=\frac{R_{i}(X, Z)}{S_{i}(X, Z)}$ (resp. $f_{i+1}=\frac{R_{i+1}(X, Z)}{S_{i+1}(X, Z)}$ ) be the real rational map constructed out of $C_{i}$ (resp. $C_{i+1}$ ) as explained in section 7 Here again, the polynomials $R_{i}(X, Z)$ and $S_{i}(X, Z)\left(\operatorname{resp} \cdot R_{i+1}(X, Z)\right.$ and $\left.S_{i+1}(X, Z)\right)$ have no common factor.

According to section [8] the graph $\Gamma_{ \pm}\left(\Phi_{i}\right)$ (resp. $\Gamma_{ \pm}\left(\Phi_{i+1}\right)$ ) is either $\Gamma_{ \pm}\left(C_{i}\right)$ (resp. $\left.\Gamma_{ \pm}\left(C_{i+1}\right)\right)$ or a perturbation of this signed real rational graph in some points distinct from 0 and $\infty$. So, the gluing of $\Gamma_{ \pm}\left(\Phi_{i}\right)$ and $\Gamma_{ \pm}\left(\Phi_{i+1}\right)$ is determined by the neighborhood of $\infty$ in $\Gamma_{ \pm}\left(C_{i}\right)$ and 0 in $\Gamma_{ \pm}\left(C_{i+1}\right)$.

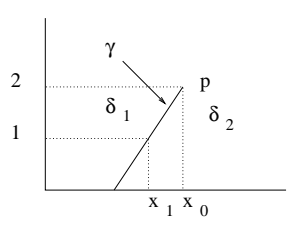

a)

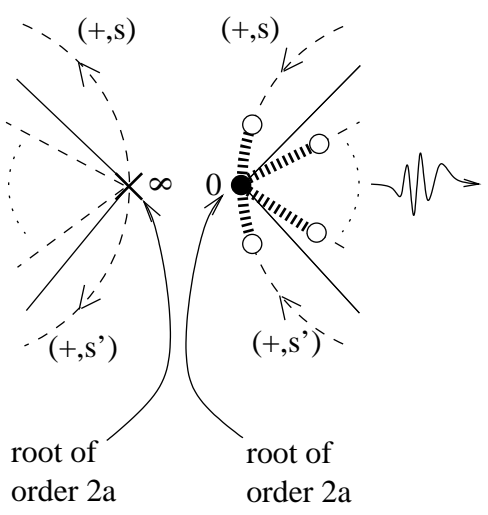

order $2 \mathrm{a}$ order $2 \mathrm{a}$

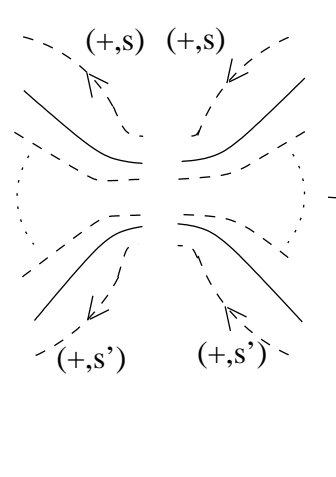

b)

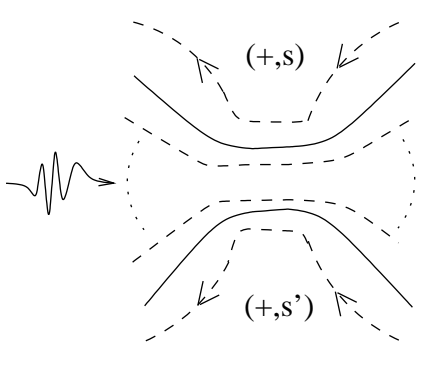

Figure 17:

A simple calculation shows that

- if $x_{1}$ is an integer, then $\infty$ (resp. 0$)$ is a root of order $2\left(2 x_{0}-x_{1}\right)$ of $R_{i}-S_{i}$ (resp. $\left.R_{i+1}\right)$,

- if $x_{1}$ is not an integer, then $\infty$ (resp. 0 ) is a root of order $2\left(2 x_{0}-\left[x_{1}\right]\right)-1$ of $R_{i}-S_{i}$ (resp. $\left.R_{i+1}\right)$,

where $[x]$ denote the integer part of the real $x$.

It follows from the construction of $\Gamma_{ \pm}\left(\Phi_{i}\right)$ and $\Gamma_{ \pm}\left(\Phi_{i+1}\right)$ and from condition (1) of section 3 that the pair of signs on the positive (resp. negative) part of $\Gamma_{ \pm}\left(\Phi_{i}\right)$ near $\infty$ and $\Gamma_{ \pm}\left(\Phi_{i+1}\right)$ near 0 also coincide.

Let $U_{0}$ be the connected component of $f_{i+1}^{-1}([\infty, 0])$ which contains 0 . Then cut $U_{0}$ from $\Gamma_{i+1}$, cut $\infty$ from $\Gamma_{i}$ and glue it in the only possible way, as it is depicted on Figure 17b) in a particular case.

\section{Proof of Theorem 6.2}

Let $\Pi$ be a trigonal patchwork of degree $n$ satisfying conditions (1) and (2) of section 3 In this section, we show that the signed real rational graph $\Gamma_{ \pm}(\Pi)$ constructed in section 9 out of $\Pi$ corresponds to a $\mathcal{L}$-nonsingular real trigonal algebraic curve in $\Sigma_{n}$ which realizes the sign array of the corresponding patchwork.

First, we check that $\Gamma_{ \pm}(\Pi)$ is the signed real rational graph of a $\mathcal{L}$-nonsingular real trigonal algebraic curve in $\Sigma_{n}$. The only non-trivial part is to prove that the degree of $\Gamma_{ \pm}(\Pi)$ is $6 n$. In order to compute it, we count the number of preimages of 1 . So we first have to count how many preimages of 1 each element of $\theta$ brings to $\Gamma_{ \pm}(\Pi)$. According to section 9 this is equivalent to count how many preimages of 1 are lying on $\Gamma\left(\Phi_{i}\right) \backslash\{0, \infty\}$ for all element $\Phi_{i}$ of $\theta$. 

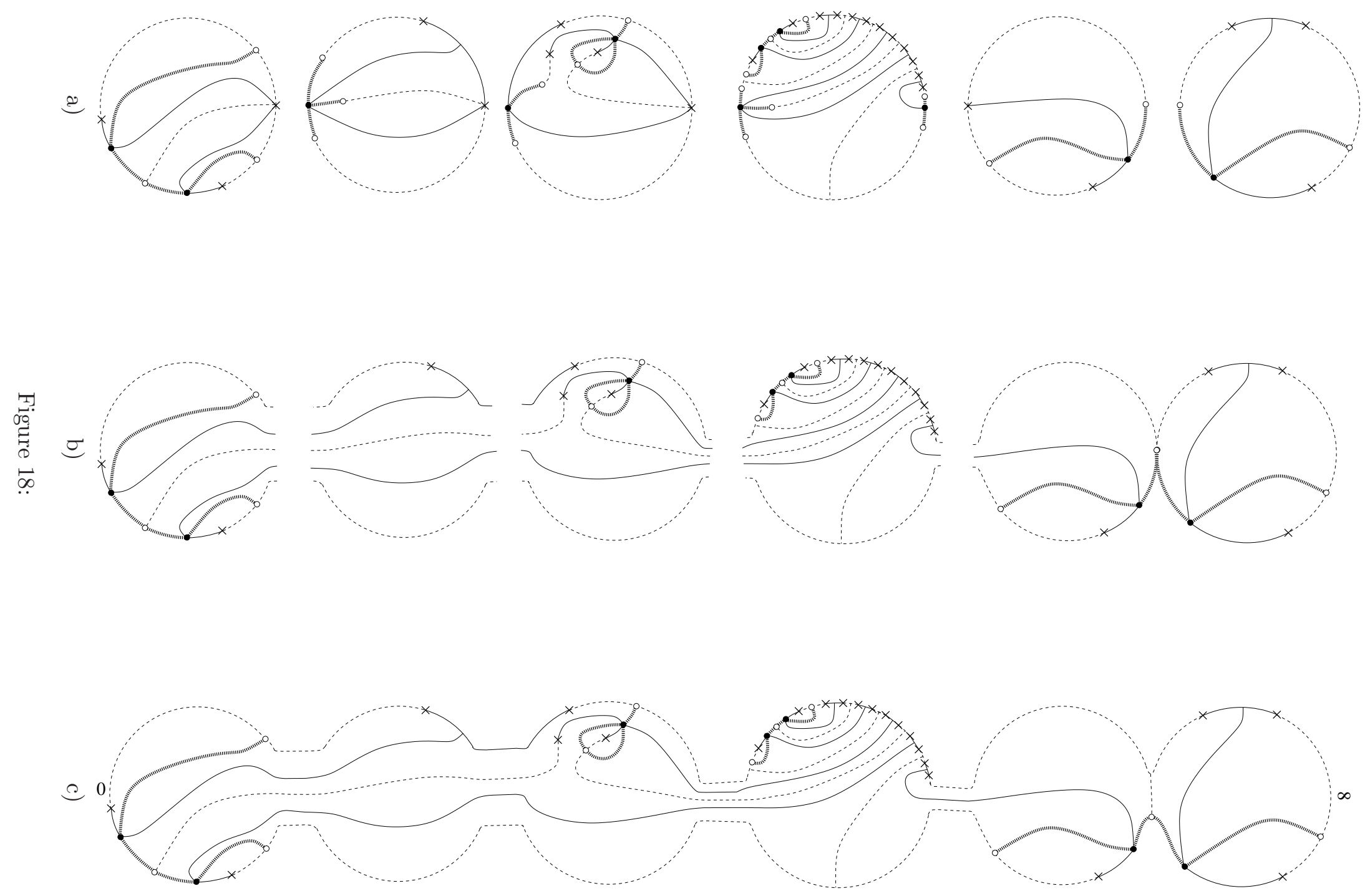
First, we count the number of roots in $\mathbb{C}^{*}$ of the discriminant of a curve. Given a convex polygon $\delta$, the quantity $I(\delta)$ is the number of integer points in the interior of $\delta$ and $\partial(\delta)$ denotes the number of integer points of the boundary of $\delta$ which have neither a maximal nor a minimal ordinate.

Lemma 10.1 Let $C(X, Y)$ be a totally nondegenerate curve with Newton polygon $\delta$. Then, the discriminant of $C(X, Y)$ has $2 I(\delta)+\partial(\delta)$ roots in $\mathbb{C}^{*}$ counted with multiplicity.

Proof : Let us consider the closure of $C(X, Y)$ in $\mathbb{C} P^{2}$ and the rational map $\pi: \mathbb{C} P^{2} \rightarrow \mathbb{C} P^{1}$ given by $\pi([x: y: z])=[x: y]$. It is well known that the genus of the normalization of $C$ is $I(\delta)$. So, applying the Riemann Hurwitz formula to the restriction of $\pi$ to $C$, we have

$$
2-2 I(\delta)=2 d-r_{1}-r_{2}
$$

where $d=\operatorname{deg}_{Y}(C), r_{1}$ is the number of roots (counted with multiplicity) in $\mathbb{C}^{*}$ of the discriminant of $C(X, Y)$ and $r_{2}$ is the sum of the tangency order of local branches of $C$ along $\{X=0\}$ and $\{Z=0\}$. As $C(X, Y)$ is totally nondegenerate, we have $r_{2}=2 d-\partial(\delta)-2$, so

$$
r_{1}=2 I(\delta)+\partial(\delta)
$$

The Lemma is proved.

Corollary 10.2 The real rational graph $\Gamma_{ \pm}(\Pi)$ constructed out of a trigonal patchwork of degree $n$ is of degree $6 n$.

Proof: It is clear from the construction and the hypothesis on the trigonal patchwork that the degree of $\Gamma_{ \pm}(\Pi)$ is equal to the sum of the numbers of preimages of 1 in $\Gamma\left(\Phi_{i}\right) \backslash\{0, \infty\}$ for all $\Phi_{i}$ in $\theta$. From section 8 and Lemma 10.1 we know that this number is equal to $2 I(\Delta)+\partial(\Delta)$ which is equal to $6 n$.

Proposition 10.3 The signed real rational graph $\Gamma_{ \pm}(\Pi)$ is realizable by a $\mathcal{L}$-nonsingular real algebraic trigonal curve $C$ in $\Sigma_{n}$.

Proof : The degree of $\Gamma_{ \pm}(\Pi)$ is given by Corollary 10.2 and all the other conditions of Proposition 7.7 are satisfied by construction.

Choose a standard coordinate system on $\Sigma_{n}$ such that the point $\infty$ on $\Gamma_{ \pm}(\Pi)$ corresponds to the fiber at infinity and let $S A_{C}$ be the sign array of the curve $C$ in this coordinate system.

Proposition 10.4 Under the hypothesis (1) and (2) of section 3 , the sign array $S A_{C}$ and the sign array constructed out of $\Pi$ in section 4.2 coincide.

Proof : Let $\left[s_{0}, s_{1} \ldots s_{u}\right]$ (resp. $\left[t_{0}, t_{1} \ldots t_{v}\right]$ ) be the sign array $S A_{C}$ (resp. associated to the patchwork). First note that a direct consequence of sections [6 8 and 9 is that $u=v$, i.e. both curves have the same number of tangency points with the pencil of vertical lines. Hence, to prove Theorem 6.2. we have just have to prove that $s_{j}=t_{j}$ for any $j$.

It is clear from sections [6. 8 and 9 that both $s_{j}$ and $t_{j}$ come from the same real $x_{j}$ for some $\Phi_{i}$. Choose a real rational map $f=\frac{-4 P^{3}}{27 Q^{2}}$ which realize $\Gamma_{ \pm}(\Pi)$.

If $\Phi_{i}$ contains no polygon with a horizontal edge of height 2 , then $x_{j}$ corresponds to a root of the discriminant of a curve of $\Pi$ whose Newton polygon is in $\Phi_{i}$. Hence, $s_{j}$ which is the sign of $Q\left(x_{j}\right)$, 
is the second element of the pair of signs which labels $\Gamma_{ \pm}\left(\Phi_{i}\right)$ near $x_{j}$. But according to section [ 6 . this is also $t_{j}$.

If $\Phi_{i}$ contains a polygon with a horizontal edge of height 2 , denote by $a_{1}(X)$ the polynomial corresponding to this edge. Let $a$ be the number of roots of $a_{1}(X)$ counted with multiplicity which are strictly between 0 and $x_{j}$ and let $\sigma$ be the sign of $a_{1}(x)$ for $x \in \mathbb{R}^{*}$ small enough with the same sign as $x_{j}$. There are two possibilities, each of them can checked directly from the construction of $\Gamma_{ \pm}\left(\Phi_{i}\right)$.

- If $x_{j}$ is not a root of $a_{1}(x)$, then $s_{j}=(-1)^{a} \sigma=t_{j}$.

- If $x_{j}$ is a root of $a_{1}(x)$, then $x_{j}$ gives rise to two elements of both sign arrays, say $s_{j}, s_{j+1}, t_{j}$ and $t_{j+1}$. We have $s_{j}=-(-1)^{a} \sigma=t_{j}$ and $s_{j+1}=(-1)^{a} \sigma=t_{j+1}$.

So in any case, we have $s_{j}=t_{j}$.

Example : One can check on Figure 18 ), that the obtained real rational graph corresponds to a non singular real algebraic trigonal curve realizing the sign array of the patchwork depicted in Figure 3

\section{Remark on an equivariant version Theorem 3.4}

We were guided in this work by the following observation : the position of the real part of a nonsingular real algebraic curve in $\Sigma_{n}$ with respect to the pencil of lines is totally encoded by the sign array realized by some collection of polynomials in one variable, namely the leading coefficients of some signed subresultant sequences (see BPR03] or [Hon for example). It seems to us that this observation could lead to interesting results in real algebraic geometry, especially in the study of the need of convexity in patchworking.

In the case of trigonal curves, the polynomials whose sign array encode the topology of the real part of the curve are exactly, with notation of section 4 the polynomials $P(X), Q(X)$ and $D(X)$. Moreover, such three polynomials with a prescribed sign array can be constructed using signed real rational graphs.

On the other hand, real rational graphs carry much more informations than the knowledge of the topology of the real part of real algebraic curves. Indeed, one can extract the position with respect to the pencil of lines of the complexification of any real trigonal curve out of its associated real rational graph. Hence, one can consider not only isotopies of curves in the real part of the surface $\Sigma_{n}$ but also equivariant isotopies of curves in $\Sigma_{n}$. That means that it is possible to state an equivariant version of Theorem 3.4 and Theorem 6.2 .

Proving such an equivariant statement would carry us quite far away from our original framework. So we only give the main ingredients needed in its formulation and in its proof.

Step 1. In the patchwork construction, one can use complexification of lattice polygons and complex equivariant charts of real polynomials as defined in IS02, IS03 and Vir. Then, the patchwork procedure gives a piecewise smooth surface, invariant under the action of the complex conjugation, in the complexification of the triangle with vertices $(0,0),(0,3)$ and $(3 n, 0)$.

Step 2. One can use the braid monodromy factorization (see KT00 or KK03 for example) to encode the position of a complex algebraic curve in $\Sigma_{n}$ with respect to the pencil of lines. An equivariant version of this encoding, based on previous papers by Orevkov (see Ore99. for example), should be used to deal with real algebraic curves. 
Step 3. As we associated a sign array to a trigonal patchwork (in section 6), one can associate in a similar way an equivariant braid monodromy factorization to an equivariant trigonal patchwork (defined in Step 1).

Step 4. One can extract the equivariant braid monodromy factorization of the real trigonal algebraic curve constructed in section [10 out of the signed real rational graph constructed in section 9 and prove that it coincides with the one constructed in Step 3.

\section{References}

[Ber06] B. Bertrand. Asymptotically maximal families of hypersurfaces in toric varieties. Geom. Dedicata., 118(1):49-70, 2006.

[Bih] F. Bihan. Asymptotiques de nombres de Betti d'hypersurfaces projectives réelles. Preprint arXiv: math.AG/0312259, (French).

[BPR03] S. Basu, R. Pollack, and M. F. Roy. Algorithms in real algebraic geometry, volume 10 of Algorithms and Computation in Mathematics. Springer-Verlag, Berlin, 2003.

[Bru] E. Brugallé. Symmetric plane curves of degree 7 : pseudo-holomorphic and algebraic classifications. arXiv : math.GT/0404030.

[Bru06] E. Brugallé. Real plane algebraic curves with asymptotically maximal number of even ovals. Duke Math. J., 131(3):575-587, 2006.

[CH80] R. Connelly and D. W. Henderson. A convex 3-complex not simplicially isomorphic to a strictly convex complex. Proc. Camb. Phil. Soc., 88(2):299-306, 1980.

[dLW98] J. A. de Loera and F. J. Wickiln. On the need of convexity in patchworking. Adv. in Appl. Math., 20:188-219, 1998.

[Haa95] B. Haas. Les multilucarnes: nouveaux contre-exemples à la conjecture de Ragsdale. C. R. Acad. Sci. Paris Sér. I Math., 320(12):1507-1512, 1995. (French).

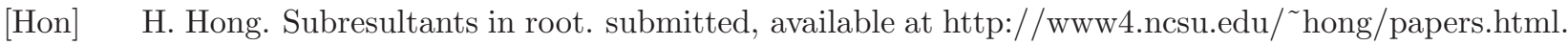

[IS02] I. Itenberg and E. Shustin. Combinatorial patchworking of real pseudo-holomorphic curves. Turkish J. Math., 26(1):27-51, 2002.

[IS03] I. Itenberg and E. Shustin. Viro theorem and topology of real and complex combinatorial hypersurfaces. Israel J. Math., 133:189-238, 2003.

[Ite93] I. Itenberg. Contre-exemples à la conjecture de Ragsdale. C. R. Acad. Sci. Paris Sér. I Math., 317(3):277-282, 1993. (French).

[Ite01] I. Itenberg. On the number of even ovals of a nonsingular curve of even degree in $\mathbb{R} P^{2}$. In Topology, ergodic theory, real algebraic geometry, volume 202 of Amer. Math. Soc. Transl. Ser. 2, pages 121129. Amer. Math. Soc., Providence, RI, 2001.

[IV] I. Itenberg and O. Ya. Viro. Maximal real algebraic hypersurfaces of projective spaces. in preparation.

[KK03] V. S. Kulikov and V. M. Kharlamov. On braid monodromy factorizations. Izv. Ross. Akad. Nauk Ser. Mat., 67(3):79-118, 2003.

[KT00] V. S. Kulikov and M. Taukher. Braid monodromy factorizations and diffeomorphism types. Izv. Ross. Akad. Nauk Ser. Mat., 64(2):89-120, 2000.

[LdM] L. Lopez de Medrano. Courbure totale des variétés algébriques réelles projectives. Thèse doctorale, 2006, (French). 
[NSV02] S. Natanzon, B. Shapiro, and A. Vainshtein. Topological classification of generic real rational functions. J. Knot Theory Ramifications, 11(7):1063-1075, 2002.

[Ore99] S. Yu. Orevkov. Link theory and oval arrangements of real algebraic curves. Topology, 38(4):779-810, 1999.

[Ore03] S. Yu. Orevkov. Riemann existence theorem and construction of real algebraic curves. Annales de la Faculté des Sciences de Toulouse, 12(4):517-531, 2003.

[Ris92] J. J. Risler. Construction d'hypersurfaces réelles (d'après Viro). Séminaire Bourbaki, 763, 1992. (French).

[San] F. Santos. Unpublished.

[Shu99] E. Shustin. Lower deformations of isolated hypersurface singularities. Algebra i Analiz, 11(5):221$249,1999$.

[Vir] O. Ya. Viro. Patchworking real algebraic varieties. http://www.math.uu.se/ oleg.

[Vir84] O. Ya. Viro. Gluing of plane real algebraic curves and constructions of curves of degrees 6 and 7. In Topology (Leningrad, 1982), volume 1060 of Lecture Notes in Math., pages 187-200. Springer, Berlin, 1984.

[Vir89] O. Ya. Viro. Real plane algebraic curves: constructions with controlled topology. Leningrad Math. J., 1(5):1059-1134, 1989.

\section{Benoit Bertrand}

Section de mathématiques

Université de Genève

case postale 64 ,

2-4 rue du Lièvre,

Genève

Suisse

E-mail : benoit.bertrand@math.unige.ch

\section{Erwan Brugallé}

Max Planck Institute für Mathematik

Vivatsgasse, 7

D-53111 Bonn

Deutschland

E-mail : brugalle@mpim-bonn.mpg.de 